Cahiers d'études africaines

\title{
Pactes, alliances et plaisanteries
}

Pratiques locales, discours global

\section{Cécile Canut et Étienne Smith}

\section{CpenEdition}

\section{Journals}

Édition électronique

URL : http://journals.openedition.org/etudesafricaines/6198

DOI : 10.4000/etudesafricaines.6198

ISSN : 1777-5353

Éditeur

Éditions de l'EHESS

Édition imprimée

Date de publication : 1 décembre 2006

ISBN : 978-2-7132-2129-3

ISSN : 0008-0055

Référence électronique

Cécile Canut et Étienne Smith, « Pactes, alliances et plaisanteries », Cahiers d'études africaines [En ligne], 184 | 2006, mis en ligne le 08 décembre 2006, consulté le 19 avril 2019. URL : http:// journals.openedition.org/etudesafricaines/6198; DOI : 10.4000/etudesafricaines.6198

Ce document a été généré automatiquement le 19 avril 2019

(c) Cahiers d'Études africaines 


\title{
Pactes, alliances et plaisanteries
}

\author{
Pratiques locales, discours global
}

\author{
Cécile Canut et Étienne Smith
}

"A unique Malian cultural custom known as "cousinage" provides a basis for understanding among these ethnic groups. This open and friendly acknowledgment of differences seems to provide Malians a sense of confidence in their common nationhood while defying African interethnic stereotypes $»^{1}$.

"C'est au mérite du professeur de toucher du doigt un des aspects de cette “ exception guinéenne" dont on a souvent parlé. [...] la vraie particularité de la Guinée : l'existence d'un pacte national non écrit mais qui fait que les Guinéens soient si tolérants et hospitaliers malgré les difficultés de toutes sortes. [...] ce pacte non écrit a été conçu au temps de l'empire du Mali, au treizième siècle [...]. C'est Soundiata Keita et ses illustres successeurs [...] qui ont conçu ce concept de "pacte social" à travers le système bien élaboré du Sanankuya $»^{2}$.

"Nous devons tous, notamment ceux d'entre nous qui constituent la classe politique, entretenir cette flamme qui fait rayonner notre cher pays, qui a toujours su contenir ses frissons et ses crises, à l'unisson et avec intelligence : c'est cela que d'aucuns dénomment "l'exception sénégalaise", faite de tolérance et de respect de l'autre. Pour situer ces valeurs, j'ai beaucoup interrogé l'histoire et il m'a paru finalement plus simple de retenir qu'elles résident, peut-être, dans le charme de notre cousinage à plaisanterie, si ce n'est dans le fruit de l'ambiance sous l'arbre à palabres où ont toujours prévalu le pacte de confiance et l'éthique de la parole donnée $»^{3}$.

«Le Burkina Faso, il faut le dire, est un pays "atypique" [...]. Un autre atout, pour ne pas dire secret, est la parenté à plaisanterie, véritable ciment social qui unit plusieurs ethnies de notre pays. C'est là une chance culturelle inouïe $»^{4}$.

«Malgré la multiethnicité qui le caractérise, le Niger est l'un des rares pays en Afrique où règnent, au sein de la population, une symbiose, une harmonie et un respect mutuel. Des liens de cousinage à plaisanterie, tissés depuis des temps immémoriaux entre les ethnies, font que les tensions et les crises éventuelles sont automatiquement et socialement atténuées dès lors que l'arme du cousinage à plaisanterie est brandie $»^{5}$.

"Most Zambians have joking relations with other tribes; the relationships go back many years. [...] this is an important distinction from other countries, where greater animosity exists. Zambians may consider their tribe superior to another, but there is an overall sense of unity across all groups $»^{6}$. 
1 La notoriété nouvelle du thème des « relations à plaisanterie ", ces dernières années, dans l'espace public de plusieurs pays d'Afrique de l'Ouest méritait à tout le moins d'être questionnée. Ce fut l'objectif du colloque Alliances à plaisanteries et politiques en Afrique de l'Ouest qui a réuni anthropologues, politistes et sociolinguistes ${ }^{7}$. Si ces fameuses relations à plaisanterie ont été un temps un objet "classique» de l'anthropologie, il était nécessaire de renouveler les questionnements sur cet ensemble de pratiques qui ont, à un moment ou un autre, interpellé les chercheurs sur des terrains différents. Cet objectif résultait d'un triple constat.

2 - Le retour critique, déjà ancien, sur certains topoi de la «bibliothèque coloniale» (Mudimbe 1988) telle que la notion de "tribu» (Vail 1989), «ethnie» (Amselle \& M’Bokolo 1985 ; Chrétien \& Prunier 1989), ou « caste » (Conrad \& Frank 1995) avait jusque récemment (Fouéré 2004) épargné le cas des "relations à plaisanterie », en raison sans doute de son inactualité tant scientifique que politique, contrastant avec l'actualité des disputes éthiques périodiquement relancées autour des vieux meubles du «tribalisme» et de l'« ethnicité ».

3 - La déconstruction du paradigme fonctionnaliste largement opérée dans les sciences sociales n'avait pas non plus atteint les rivages de cet objet "parenté à plaisanterie " - des études récentes prolongeaient même plus que de raison ses vieux jours - alors même que sa déconstruction offrait des opportunités d'analyses renouvelées des « alliances à plaisanterie ».

4 - L'actualisation du thème lui-même devait finalement connaître un essor étonnant dans l'espace politico-médiatique, résultant d'une conjonction de facteurs dont il faut rendre compte, et dont l'émergence d'un discours réactif ou « dérivé », entendant inverser le portrait ethniciste et négatif fait de l'Afrique, n'est pas le moindre.

5 Ainsi donc, sur la base de ce constat, se justifie une série d'analyses qui, dans leur globalité, rendent compte à la fois des pratiques actuelles, sans cesse réinventées par les individus dans leur vie quotidienne, et de la multiplication des mises en discours de ces pratiques dans les espaces publics "africains $»^{8}$ actuels qui empruntent précisément à deux matrices déjà mises en question par ailleurs: l'ethnologique culturaliste de la «bibliothèque coloniale » et l'irénisme du fonctionnalisme.

6 L'analyse de ces deux dimensions, pratique et discursive, se veut donc une interrogation des enjeux éminemment politiques et contemporains de ces alliances à plaisanterie, afin de sortir de l'ornière de la réification et de la déshistoricisation de ces pratiques, souvent inconscientes, opérées à des fins louables, mais souvent illusoires, d'ingénierie « culturelle » du social et de « pacification » des sociétés.

Les mots et les choses

7 Une des difficultés de l'exercice résidait dans l'adéquation entre « le mot » et « la chose ». Une fois la critique du concept classique de " parenté à plaisanterie » opérée, l'important est de constater la variété des pratiques dont il n'est pas toujours certain qu'elles doivent se laisser réduire à un terme générique. Au sein d'un continuum de pratiques sociales observables, lesquelles ranger sous l'étiquette savante de "relations à plaisanterie »? Cette étiquette elle-même n'est-elle pas vaine comme le soulignaient déjà avec amusement Marcel Griaule (1948), Mary Douglas (1968) et Mary Tew (1951) ? Tant au plan de la spécification des groupes et individus impliqués dans la relation - relations entre cousins croisés, oncles maternels et neveux utérins, petits-enfants et grands-parents, beaux-frères et belles-sœurs, classes d'âges alternées, matriclans, patriclans, groupes 
socioprofessionnels, maîtres et captifs, quartiers, villages, contrées, entités politiques, labels patronymiques, noms d'honneurs, groupes désormais «ethniques»-, que de l'intensité de la relation connexe - alliance matrimoniale préférentielle ou interdit de mariage, pacte de sang réel ou pacte de sang métaphorique, simples moqueries ou relation sanctionnée par une "amende", liberté de ton complète ou plaisanteries minimales, humour gratuit ou service attendu -, ou de son orientation - de la symétrie à l'asymétrie -9 , c'est indiscutablement l'hétérogénéité qui caractérise cet ensemble de relations qui ont, tour à tour, été versées dans le champ, décidément trop vaste, des «relations à plaisanterie ». De fait, la réponse à la question «qu'est-ce que c'est?» suppose déjà un corps constitué de connaissances dont il a pu être montré dans quelle mesure il reposait sur une «abstraction d'abstractions » (Fouéré 2004 : 67). Plutôt que de fixer uniformément les limites des relations à étudier, il a paru plus judicieux de laisser les auteurs libres de les déterminer. Malgré tout, l'ensemble des travaux présentés ici se concentrent sur les relations hors du domaine de la famille afin de focaliser les analyses sur les relations entre groupes plus vastes (labels patronymiques, communautés), quitte à repérer naturellement que la métaphore de ce premier domaine de la «famille» et du « cousinage » au sens strict se retrouve dans ces relations plus étendues ${ }^{10}$.

8 Une fois rejetée, l'exigence de définition stricte incarnée par la question « qu'est-ce que c'est?", restait, au minimum, à se mettre d'accord sur une autre question, "de quoi parle-t-on?». Mais surgissait alors la question de la variété des termes vernaculaires dont la liste n'est jamais exhaustive. Des études concrètes et contextualisées doivent d'ailleurs souligner l'entrelacs des significations, les possibilités de déclinaisons entre différents types de relations à plaisanterie ("amère " et "douce ", "légère » et "blessante", "forte " et "faible » etc.), et leurs différents niveaux de formalisation pactuelle (pactes $v s$ simples taquineries) attestés par le vocabulaire. Il est donc apparu préférable de conserver les termes employés par les locuteurs en situation ${ }^{11}$.

9 La tradition écrite (l'anthropologie classique) est tout aussi riche en termes qui se veulent génériques, dont deux se sont petit à petit imposés comme concepts : «joking relationship 》 et «parentés à plaisanterie ». Mais ceux-ci ne doivent pas faire oublier les synonymes et expressions analogues en français comme en anglais qui ont balisé la mise en discours savant de ces pratiques ${ }^{12}$. Ces deux termes canoniques étant critiquables à maints égards, nous avons laissé les auteurs libres de l'emploi des termes savants ${ }^{13}$.

Le concept de "parenté à plaisanterie » ou "joking relationship $»^{14}$ a donc une historicité qu'il faut mettre en évidence. Des pratiques ont bel et bien été décrites, lesquelles ont été nommées par les acteurs en situation et subsumées par les «passeurs » et les ethnologues dans la catégorie générale des "parentés à plaisanterie ». Des prototypes fameux, comme la relation Peuls/forgerons (Doumbia 1936) ou Dogon/Bozo (Griaule 1948), ainsi que l'énumération de propriétés caractéristiques de ces relations dans une optique comparative (Mauss 1928; Radcliffe-Brown 1940) ont balisé le développement de ce champ d'études dont il faut évoquer ici brièvement les étapes.

La canonisation de la tradition, acte 1

«Il existe entre certaines de ces familles des sortes de servitudes qui rappellent un peu celles de la féodalité : ainsi un Diakité peut tirer la barbe d'un Dialos sans que celui-ci soit en droit de s'en formaliser. D'autres, ce sont, je crois, les Diaras et les Traourés, se font, en guise de salut, des questions baroques sur l'état plus ou moins florissant de leur virilité » (Béchet 1889 : 187).

Des pratiques observées à la mise en discours générique 
11 Le premier contact entre les voyageurs étrangers, conquérants ou administrateurs coloniaux et les pratiques qui seront par la suite labellisées " parentés à plaisanterie »se fait sous le signe de l'étonnement, parfois mêlé de fascination, pour ces «bizarres façons » et « usages très particuliers » (Béchet 1889 : 186-187), pour une coutume «bien étrange » (Reichar 1890 cité par Fouéré $2004: 38)^{15}$, ce "phénomène très particulier » (Delafosse 1912 vol. 3 : 106) ou cette «relation curieuse» (Labouret 1934: 100). À ce premier stade de l'«africanisme», la mention d'alliances apparaît aussi dans les recensions de traditions orales ou certaines monographies en Afrique de l'Ouest (Boilat 1853 : 179 ; Tautain 1885 : 8 ; Monteil 1903 : 320, 1915 : 281, 1924 : 225 ; Desplagnes 1907 : 91 ; Arnaud 1921 : 261 ; Meek 1925 ; Wilson-Haffenden 1930 : 117-125 ; Doumbia 1936), ou en Afrique australe (Dale 1896; Mac Alpine 1906: 257-268; Junod 1912 ; Smith \& Dale 1920 : 295s ; Goodall 1921 : 75 ; Melland 1923 : 251-253 ; Doke 1931 : 197 ; Young 1931 : 169 ; Brelsford 1935 : 212) et orientales (Abdy 1924 ; Mc Vicar 1935 ; Scrivenor 1937).

Les dénominations locales sont donc conservées, que ce soit utani au Tanganyika, banungwe en Rhodésie et senankuya au Soudan français. Les auteurs se contentent généralement de simples descriptions. Si Delafosse (1912 vol. 3 : $106 \mathrm{~s}$ ) n'est donc pas le premier à décrire les senankuya observées au Soudan français (Béchet 1889 ; Monteil 1903), son Haut-Sénégal Niger en fournira en revanche la première analyse générale, dépassant la présentation de prototypes précis, bien qu'il n'échafaude pas un terme générique. Cette analyse des senankuya entre jamuw, constituera le premier maillon de la chaîne d'intertextualité sur le sujet pour l'Afrique de l'Ouest francophone (Humblot 1918 ; Labouret 1929 ; Pageard 1958).

Mais le terme générique "joking relationship » sera d'abord forgé sur les terrains nordaméricains par Lowie (1912: 204-206, 1917 : 42-45, 79-80) et repris ensuite par Radin (1923 : 85). On apprend d'ailleurs par Marcel Mauss (1928: 7) que «les observateurs américains ont été très frappés de la singularité de ces usages ». C'est Mauss qui sera l'initiateur d'une théorisation générale transversale, lors d'une conférence donnée en 1926 à l'École pratique des hautes études et publiée deux ans plus tard, dans laquelle il traduit le terme anglais utilisé par Lowie - «joking relationship»- par "parenté à plaisanterie ». Mauss souligne l'importance de ces « parentés à plaisanterie », qui seraient l'envers des relations d'évitement et de respect («les parentés à respect») dues à certaines catégories précises de parents, sorte de défoulement du poids de l'étiquette: «Ces institutions ont une fonction fort claire. [...] elles expriment un état sentimental psychologiquement défini : le besoin de détente, un laisser-aller qui repose d'une tenue par trop compassée. » Et Mauss de comparer cette fonction de détente à des «soldats échappant à la position sous les armes", des "écoliers s'égaillant dans la cour du collège » et des "messieurs se relâchant au fumoir de trop longues courtoisies vis-à-vis des dames » (ibid. : 8-9). Cependant il ne se satisfait pas complètement de cette hypothèse car il reste alors à élucider la question centrale à ses yeux : pourquoi x plaisante avec $\mathrm{Y}$ et non avec $z$ ? La réponse doit se trouver, selon lui, dans le système de parenté et dans les positions respectives de $\mathrm{X}, \mathrm{Y}$ et $\mathrm{Z}$, requérant donc une théorie générale des parentés à plaisanterie qui permettrait d'expliquer pourquoi existent ces «opposition et solidarité mélangées et alternées » (ibid. : 10). Il propose donc de ranger ces parentés à plaisanterie dans la catégorie des "systèmes de prestations totales " qui organisent la coopération entre groupes de parents et alliés par les «rivalités obligatoires ». À ce compte-là, les parentés à plaisanterie, "institutions plus frustes, et plus simples ", seraient un stade antérieur des « institutions du potlatch infiniment développées » (ibid. : 12). 
14 Le terme générique est ainsi né de la volonté de classer un ensemble de pratiques similaires observées dans trois « provinces ethnographiques » du monde constituées par l'ethnologie occidentale : «la Prairie américaine », "les îles mélanésiennes » et les «tribus africaines» (ibid.: 6). Dès lors, on assistera à la subsomption progressive des pratiques observées sur le terrain sous ce terme commun, abstraites des contextes sociopolitiques et des situations concrètes d'interlocution. Les pratiques de plaisanterie un tant soi peu ritualisées ne pourront plus être observées sans être labellisées et importées dans le champ de la "parenté à plaisanterie ». Si les deux autres "provinces ethnographiques » donnent toujours lieu à des observations de ces pratiques (Océanie : Hocart 1923, 1935 ; Leenhardt 1930; Mead 1934; Thompson 1937 ; Amérique du Nord : Eggan 1937: 75-81), c'est la troisième qui verra se développer les études les plus nombreuses sur le sujet. En effet, un an après la publication de la conférence de Mauss, Henri Labouret (1929) introduit dans la revue Africa le terme de "parenté à plaisanterie » à propos de relations observées au Sénégal, au Soudan français et en Haute-Volta, traduisant les relations de senankuya (manding), dendiraagu (pulaar) et gàmmu (wolof) par ce terme générique qu'il applique à la fois aux relations asymétriques entre certaines catégories de parents (cousins croisés), entre clans et entre peuples ${ }^{16}$. Ce ne sera pas la fin des étiquettes alternatives évoquées plus haut, mais la canonisation effective du terme français par la revue Africa, repris ensuite par Paulme (1939) et posé comme l'équivalent définitif du terme anglais "joking relationship ». Ce terme est introduit en Afrique de l'Est par Mc Vicar (1935) qui traduit le terme swahili utani par «joking relationship », puis repris par Radcliffe-Brown (1940), ainsi que Pedler (1940) et Moreau (1941, 1944). Entre les essais théoriques de Mauss (1928) et de Radcliffe-Brown (1940, 1949), le champ s'est enrichi de nouvelles études de terrain (Evans-Pritchard 1929, 1933 ; Richards 1935, 1937 ; Fortes 1945, 1949) qui servent de matériau ethnographique à Radcliffe-Brown (1924), en plus de sa propre expérience de terrain.

La théorisation de Radcliffe-Brown (1968: 182), après celle de Mauss, marque une nouvelle étape dans la généralisation, renouant avec une analyse transcontinentale et le souci de produire une théorie générale du phénomène: "La parenté à plaisanteries décrite en Afrique entre des clans et des tribus peut [...] être intégrée dans une théorie unique qui réduit tous les exemples de ces parentés à un type général de situation structurale." Comme Mauss, il souligne que les «coutumes d'évitement " et les «privilèges de plaisanterie » doivent être étudiés ensemble, car ce sont les deux façons inverses de faire tenir ensemble dans la société les forces opposées de la conjonction et de la disjonction. La relation à plaisanterie est une forme de relations d'amitié, qui est « une combinaison singulière de bienveillance et d'antagonisme ", " une relation d'amitié dans laquelle existe un antagonisme apparent, contrôlé par des règles conventionnelles ». Tout comme Mauss, Radcliffe-Brown range ces relations de plaisanterie dans la catégorie des "rivalités amicales", comparables à la relation qui lie par exemple les universités d'Oxford et de Cambridge... (ibid. : 183). Après Mauss, il est celui qui souligne avec le plus de vigueur la fonction de pacification de ces relations de plaisanterie. Dans cet «antagonisme joué » et sa "répétition régulière », l'hostilité apparente «est la contrepartie d'une amitié réelle » (ibid. : 159). Radcliffe-Brown (ibid.: 177) inaugure ainsi l'hypothèse irénique selon laquelle la « relation comportant l'échange d'insultes ainsi que l'obligation de ne pas les prendre au sérieux est la seule qui, au moyen de ces conflits simulés, évite les conflits réels ». 

Brown est critiqué, ce n'est pas pour son biais fonctionnaliste, mais pour son comparatisme. Il l'est par Marcel Griaule (1948) qui propose le terme d'«alliance cathartique " pour décrire l'alliance Bozo-Dogon, à qui Radcliffe-Brown (1949) répondra un an plus tard. Griaule reproche à Radcliffe-Brown l'idée même d'une théorie comparative des parentés à plaisanterie abstraites des cosmogonies particulières : «On a donné le nom de "parenté à plaisanteries" à des institutions et manifestations diverses qui ne sont peut-être pas de même nature [...] on a donné de l'importance à un facteur apparent, la plaisanterie injurieuse, qui se retrouve dans des occasions nombreuses et sans rapports les unes avec les autres. [...] retenir l'aspect "plaisanterie" de plusieurs phénomènes risque de lier les choses les plus hétéroclites. Une cloche sonne le glas et les mariages. Il ne viendrait à l'idée de personne de prétendre que les funérailles et les noces se rencontrent dans une série dite "cérémonies à cloches" " (Griaule 1948: 242). Pour Griaule, ce n'est pas la «jubilation dans l'injure » qui est importante dans la relation mangou, mais la fonction de purification des protagonistes, d'où le nom d'alliance cathartique.

Cependant, malgré leur opposition, les approches de Radcliffe-Brown et Griaule se retrouvent sur un certain nombre de points. Le fonctionnalisme du premier, à la recherche d'une théorie générale, rejoint le culturalisme "particularisant» du second, pour penser la "cohérence» et la «fonction» de pacification quasi systémique des « relations à plaisanterie » ou " alliances cathartiques ». Un débat similaire oppose Mary Douglas (Tew 1951 : 122) à Stefaniszyn (1950). Celle-ci, tout comme Girling (1957 : 102), souligne l'ineptie d'une «théorie des relations à plaisanterie » chère à Mauss, RadcliffeBrown et Stefaniszyn (Douglas 1968).

Malgré ces critiques initiales, l'anthropologie dispose désormais, avec Radcliffe-Brown, d'un concept et d'une théorie, organisant un champ de connaissance spécifique qui connaitra un développement considérable. Ce champ se nourrit également des études sur les autres «provinces ethnographiques » : océaniennes (Leenhardt 1947; Pouwer 1964; Luomala 1966), américaines (Brant 1948; Driver 1961: 462-463; Kennedy 1970; Brackelaire 1992, 1993) européennes ou japonaises (Girling 1957; Sykes 1966; Bradney 1957; Yoshida 2001) ${ }^{17}$. Mais l'essentiel des travaux porte sur des terrains africains (Tegnaeus 1954; Reynolds 1958), quoique de tous ces travaux africanistes, seuls ceux qui sont l'œuvre d'anthropologues ont réellement alimenté le champ comparatiste des théorisations, souvent fonctionnalistes, des relations à plaisanterie.

Les études sur l'Afrique centrale, fort nombreuses, ont notamment été attentives aux phénomènes de "funeral friendship", aux relations intrafamiliales et avunculaires (Stefaniszyn 1950, 1951; Tew 1951; de Sousberghe 1955; Wilson 1957; Gulliver 1957, 1958 ; White 1957, 1958; Douglas 1968; De Heusch 1974; White 1994). Mais les études de l'École de Manchester en Afrique australe, sont sans conteste les plus intéressantes (Colson 1968; Gluckman 1955, 1965; Mitchell 1956b; Boswell 1969; Handelman \& Kapferer 1972). Cependant si Elizabeth Colson (1953) ou Max Gluckman (1965:10, préface de Colson) soulignent, à juste titre, les liens étroits qu'entretiennent les alliances à plaisanterie avec l'espace moral du "clan », leur analyse de ces pratiques d'alliances et des réciprocités qu'elles organisent reste marquée par un souci de rationalisation et d'aplatissement en termes "d'obligations" et de valeurs morales immuables. En revanche, l'analyse de Clyde Mitchell (1956b) dans The Kalela Dance est plus novatrice, dans la mesure où elle rompt avec l'étude traditionnelle de ces pratiques en milieu rural, 
qu'il a lui-même pratiquée (Mitchell 1956a), afin de saisir leurs usages renouvelés en milieu urbain dans le cadre du "tribalism in town» (Mitchell 1956b). Par la suite, les études des alliances à plaisanterie en situations urbaines ont été plutôt rares, hormis quelques études à Dakar (Diarra \& Fougeyrollas 1969), Dar-es-Salaam (Leslie 1963 : 37-38), Kabwe en Zambie (Handelman \& Kapferer 1972) et Kumasi au Ghana (Schildkrout 1975). S'inspirant des travaux de Goffman (1961), l'étude comparative de Don Handelman et Bruce Kapferer (1972) soulève notamment, à partir d'une analyse des interactions entre travailleurs bemba et ngoni, la question de l'enjeu de la définition du «cadre» de l'interaction de «joking relationship » en situation urbaine.

L'histoire des systèmes claniques dans l'Afrique des Grands Lacs donne également lieu à des précisions sur les «alliances à plaisanterie » (Jervis 1939:56; d'Hertefelt 1971:6; Mworoha 1977 : 35-38; Newbury 1991: 120-125; Chrétien 2000: 75-76), de même que l'ethnographie de pratiques d'injures et de plaisanterie au Burundi (Rodegem 1976) et au Rwanda (Freedman 1977). Les monographies ou travaux d'anthropologie de la parenté sur des terrains est-africains complètent le tableau (Ouganda : Beattie 1957, 1958 ; Sharman 1969; Kenya: Ueda \& Ueda 1975; McKay 1975: 133-135; Herlehy 1984: 305-306; Tanganyika puis Tanzanie : Spies 1943 ; Mayer 1950, 1951; Christensen 1963 ; Beidelman 1963, 1964, 1966 ; Rigby 1968 ; Landsberg 1977 ; Heald 1990) ${ }^{18}$. Après la «Copperbelt » dans les années 1950, analysée par l'École de Manchester, l'Afrique de l'Est anglophone semble prendre le relais et être à la pointe de la recherche sur les relations à plaisanterie. Se tient ainsi, en décembre 1966, au Makerere College de Kampala, une conférence entièrement dédiée aux «joking relationships» (Desaï 1966). Parallèlement, en 1974, Stephen Lucas publie à Dar-es Salaam, dans une perspective marxiste, l'étude la plus conséquente (sept volumes) sur les relations d'utani en Tanzanie à partir, notamment, des récits d'origine de ces relations dans les traditions orales.

On voit donc comment le concept de «joking relationship » ou " parenté à plaisanterie » a été à l'origine - et le demeure dans les revues et manuels d'anthropologie - un concept transculturel à l'homologation duquel les travaux africanistes ont largement contribué ${ }^{19}$. De plus, le label savant a été réapproprié par divers discours dans les sociétés africaines contemporaines, notamment en Afrique de l'Ouest, qui font parfois de ces parentés et alliances à plaisanterie une spécificité africaine, un savoir-faire culturel local, qui nécessite « explications » et « explicateurs » pour l'étranger qui n'est pas familier de ces «usages très particuliers». C'est un processus au terme duquel ces «relations à plaisanterie ", bien que non spécifiquement africaines, sont finalement parvenues à emblématiser l'« africanité ${ }^{20} »$.

L'Afrique de l'Ouest : une production continue

Très tôt, l'Afrique de l'Ouest se présente comme un espace-clé pour la recension d'alliances à plaisanterie ${ }^{21}$. Dans la lignée de Delafosse (1912) quelques administrateurs et militaires, fascinés par l'onomastique, et à la recherche d'un état-civil indigène " adéquat ", entretiennent un fort intérêt pour cet objet à partir de la recension quasi administrative des alliances et équivalences entre jamuw mandingues et plus largement ouest-africains, sans souci de théorisation (Arcin 1907; Humblot 1918; Delaforge 1932; Delmond 1945 ; Pageard 1958 ; Molinié 1959) ${ }^{22}$. Parallèlement, l'École griaulienne poursuit ses systématisations formelles des traditions orales en analysant ces alliances dans le cadre des réflexions sur la notion de personne et les relations entre cosmogonie et ordre social (Dieterlen 1951, 1955, 1959 ; Pâques 1954 ; Calame-Griaule 1954 ; Griaule 1973). Tous ces travaux ont en commun de souligner « l'importance » des alliances en question, mais 
aussi de négliger leur dimension politique au profit d'une lecture excessivement culturaliste.

À la suite des travaux « griauliens » des années 1950, l'intérêt pour le monde mandé, et en particulier pour la geste de Sunjata dont les fameuses senankuya sont partie intégrante, contribue largement au maintien de la problématique : selon une optique "cathartique " (Camara 1969), sous l'angle de la dimension «politique» ou «diplomatique » de ces relations à l'époque précoloniale (Niane 1960 ; Tamari 1991, 1993, 1997) et à travers les récits de migrations et de peuplements (N'Diaye 1970a, 1970b ; Innes 1974, 1976, 1978 ; Cissé \& Kamissoko 1988, 1991 ; Giesing 2000 ; Timmer 2000 ; Schaffer 2003). Quel que soit l'angle retenu, il apparait clairement que les senankuya ne peuvent être analysées sans tenir compte des stratifications socioprofessionnelles, des hiérarchies sociales et politiques dans la région telles qu'elles se donnent à voir dans les traditions orales. La réflexion sur ces pratiques est ainsi reconnectée aux mythes politiques de la région ouestafricaine.

Au-delà de cette référence aux passés des empires ouest-africains et à la geste mandingue, le thème est présent dans les monographies. Ces alliances font généralement l'objet d'une "section» dans les rubriques «famille», "parenté», "organisation sociale ", ou "relations de civilité » (par exemple: Balandier \& Mercier 1952: 10-12, 67-69; Leriche 1956 : 175 ; Ames 1956 : 158; Gamble 1957 : 54-55 ; Pageard 1959a, 1959b ; Nicolaisen 1963: 454-456; Ly 1966: 286-289; Pélissier 1966: 194, 659; Wane 1969: 206-219; N'Diaye 1970a : 19-21, 67, 78, 85-88 ; N'Diaye 1970b : 104-105 ; Pollet \& Winter 1972 : 191s ; Riesman 1974 : 124-127 ; Gailey 1975 : 76 ; Ba 1977 : 196-201 ; Lallemand 1977 ; Leynaud \& Cissé 1978 : 131-134 ; Schaffer \& Cooper 1980 : 50s, 85s ; Gravrand 1983 : 89-94). Le lien des alliances à plaisanterie avec les systèmes de classes d'âges et des rapports structuraux entre aînés et cadets est notamment l'objet d'investigations (Hammond 1964 ; Paulme 1968 ; Smith 1973). La dimension politique et l'historicisation de ces alliances sont de ce fait généralement absentes dans ces travaux au profit d'une analyse sociologique raccordant les alliances à plaisanterie aux grands traits de l'organisation sociale.

Parallèlement, les études d'ethnologie de la parole s'intéressant aux formes d'humour, d'insultes ou d'obscénité, traitent largement des "parentés à plaisanterie » (Sissoko 1950 ; Palau-Marti 1960 ; Zahan : 1963 ; Camara 1969 ; Irvine 1974, 1993 ; Lallemand 1975 ; Dupire 1979 ; Sow 1991 ; Schottman 1998 ; Schiavone 2001 : 41-67, 217-242 ; Douyon 1995 ; Long 2005; Wendling 2005). Enfin, dans les manuels linguistiques, les alliances à plaisanterie sont également évoquées, marquées du sceau de l'altérité et justifiant à ce titre des explications pour l'apprenant, «profane» en la matière (Girier 1996: 220-224; Leroy \& Balde 2003).

Cependant, le renouveau récent de la thématique des alliances à plaisanterie est surtout dû à des travaux insistant, plus explicitement encore que les travaux précédents, sur le rôle supposé de "pacification » des alliances à plaisanterie, prolongeant ainsi certains traits du culturalisme ethnologique et du fonctionnalisme irénique, hérités des « grands ancêtres » de l'ethnologie et savamment adaptés au nouveau Zeitgeist.

Le renouveau fonctionnaliste : irénisme et pacification

Dans les années 1990, l'intérêt pour les "relations à plaisanterie ", appréhendées comme outil de la "pacification des mœurs ", va renaître notamment à travers le développement des études sur la résolution des conflits. Dans les sciences sociales nord-américaines, cet intérêt débouche parfois sur une nouvelle discipline suscitant des recherches à propos des «mécanismes traditionnels» de résolution des conflits (Zartman 2000) reprenant à 
leur compte le biais d'analyse jural ${ }^{23}$ des travaux fonctionnalistes antérieurs et l'ethnologie culturaliste célébrée par de nombreuses institutions internationales. Réhabilitant ce qui a été constitué comme "tradition » par les générations précédentes d'ethnologues et leurs auxiliaires, ces travaux réactivent les "relations à plaisanterie ", censées jouer le rôle de "remède ». Fortement inspirée par le champ grandissant de l'expertise et des institutions internationales, l'insistance sur la dimension culturelle du développement (Poncelet 1994) donne une place de choix aux discours de valorisation des cousinages comme outils de pacification et de régulation sociale (Mariko 1990; Ndiaye 1992, 2000, 2001 ; Sessouma 1993 ; Legré Okou 1994 ; Badini 1996 ; Barké 1996 ; Diouf 1996 ; Diouf 1997; Idrissa 1997: 50 ; Diouf 1998: 61s; Konaté 1999; Faton 1999; Wilson-Fall 2000 ; Sarr 2000 ; Nyamba 2001; Doumbia 2002 ; Sissao 2002; Kouyaté 2003; Meïté 2004 ; Diall \& Traoré 1999 ; Teme 1999 ; Tanou 2003 ; Sambou 2005). Dans cette célébration des " cousinages » ou " parentés à plaisanterie ", c'est avant tout une réhabilitation et une célébration des "savoirs locaux" ou "savoirs endogènes " qui sont à l'œuvre ${ }^{24}$. Les "parentés à plaisanterie", au même titre que l'«arbre à palabres", la justice «traditionnelle ", les confréries de chasseurs ou le « retour des rois », constitueraient des remèdes aux conflits politico-identitaires ${ }^{25}$. Cette production exponentielle souligne un renouveau, notamment à partir de l'Afrique de l'Ouest francophone - mais pas seulement (Mkangi 1997; Wegru 2000; Myamba 2000, 2001) - et nécessite une analyse de son inscription dans le champ globalisé du néo-traditionalisme et du « recours culturel» (Poncelet 1994) largement analysé par ailleurs.

Dans leur ensemble, ces travaux récents font l'impasse sur la dimension hiérarchique des alliances à plaisanterie, pourtant soulignée par les premiers auteurs (Labouret 1929), et rappelée dans des travaux ultérieurs (Ly 1966 ; Wane 1969 ; Amselle 1977 ; Launay 1977 ; Tamari 1997; van Hoven 1997) ${ }^{26}$. À partir des années 1970, en effet, certains travaux d'anthropologie politique ont déjà proposé de rompre avec les approches cathartiques et fonctionnalistes antérieures, pour souligner l'historicité de ces alliances à plaisanterie, leur rapport au politique, les relations de pouvoir et les négociations et conversions identitaires dans lesquelles elles s'imbriquent (Schildkrout 1975, 1978 : 138-160 ; Amselle 1977 : 37-46 ; Launay 1977 ; Amselle 1990 : 59-61, 1996, 2001 : 198-202 ; Fay 1995, 1997 ; Giesing 2000 ; Timmer 2000). C'est dans cette optique que se situe ce numéro spécial.

Pour une approche politique et contemporaine

«Écoutez-moi bien, vous autres Sérère de Touba Toul et des environs, vous mes esclaves, je veux que désormais vous vous acquittiez dorénavant et à temps de votre taxe rurale sans broncher. C'est cela qui va permettre à la communauté rurale de verser sa contribution pour que le PNIR puisse venir chez vous et faire ici d'autres réalisations comme les maternités, écoles, foyers des femmes ou autres cantines que vous avez dans votre marché... C'est un ordre que je vous donne. Si vous n'avez pas d'argent, allez vendre vos poulets. Ou au besoin amenez-moi alors ces poulets. Comme vous [ne] daignez les consommer, donnez-les au Diola amateur de bonne chair que je suis. Je vous donnerai en contrepartie de quoi aller vous acquitter de votre taxe rurale",

ajouta le sous-préfet qui, joignant le geste à la parole, se tapa le ventre qu'il indique comme étant un "cimetière de poulets "; ceci pour mieux chahuter ses interlocuteurs qu'il n'hésita pas à taxer au passage de simples rustres ${ }^{27}$.

Poursuivant certaines études récentes allant dans ce sens (Canut 2002; Fouéré 2004, 2005 ; De Jong 2005), l'objectif des contributions rassemblées au sein de ce volume réside dans la production d'analyses contextualisées des «alliances à plaisanterie » selon leur 
dimension politique et contemporaine, de leurs usages renouvelés et de leur mises en discours. Sans prétendre énoncer une théorie générale comme l'auraient voulu Mauss et Radcliffe-Brown, il s'agit d'initier une réflexion comparative tant sur l'inscription de ces pratiques dans des contextes sociaux en transformation (ville, administration, vie politique, médias, mais aussi zones rurales soumises aux transformations globales) que sur l'émergence de discours politiques sur ces pratiques dans plusieurs pays de l'ouest africain. Cette comparaison est d'autant plus utile que, malgré la spécificité des contextes sénégalais, malien ou burkinabè par exemple, ceux-ci sont affectés, ces dernières décennies, par un même cadre de transformation des scènes politiques africaines: "démocratisations " sous ajustement, décentralisation, prolifération des ONG et des programmes d'institutions internationales promouvant la dimension culturelle du développement, libéralisation des espaces publics et des constructions identitaires qui constituent autant d'opportunités d'émergence de sites d'énonciation et de prises de parole. Outre ces processus de fond et leur fragilité, l'actualité du continent et en particulier trois événements majeurs qu'ont été le génocide au Rwanda et ses répercussions dans l'Afrique des Grands Lacs, la matrice des conflits de type Mano (Libéria, Sierra Leone) et enfin les dynamiques de conflit en Côte-d'Ivoire, sont à l'origine d'une réflexion sur les modes d'intégration souhaitables dans le cadre des États-nations voire au-delà ${ }^{28}$.

31 C'est à l'aune de ces contextes et de leurs conséquences sur les pratiques et discours des alliances à plaisanterie qu'il importait de s'interroger. Trois axes sont explorés tour à tour : les dynamiques de conflit et d'accommodation dans les interactions entre groupes ou individus alliés à plaisanterie, la reproduction et l'adaptation des pratiques dans les nouveaux espaces publics, et enfin les mises en discours néo-traditionalistes aboutissant à une nouvelle canonisation de la « tradition ».

Pouvoirs, mémoires et identités dans l'interaction

Depuis déjà plus d'une quinzaine d'années, des études désormais classiques ont établi l'importance des dynamiques de frontières dans la production et reproduction des identités locales et des sociétés politiques (Kopytoff 1987), des «systèmes de transformations » à l'œuvre au sein de "ces chaînes de société » (Amselle 1990). Est apparue la nécessité de réinscrire ces formations politiques et identitaires dans un espace vaste, sous-régional, un « tissu social symplectique » (Meillassoux 1978 : 132), caractérisé par la mobilité et les dynamiques d'interaction (Fay 1995, 1997; De Bruijn \& van Dijk 1997 ; Diallo \& Schlee 2000 ; Gaillard 2000 ; Kuba, Lentz \& Somda 2004). C'est donc au sein de cette matrice globale de pouvoirs (politique, militaire, économique, religieux), d'agencement des hiérarchies et de distribution des rôles qu'il faut analyser la production des alliances à plaisanterie et leurs transformations à mesure que ces contextes se modifient (Fay 1995). Il apparaît ainsi qu'historiquement une transformation de conflits politico-militaires en "alliances", constitutive de rapport de pouvoirs et de hiérarchisation, soit à l'œuvre sans qu'elle résume pour autant toutes les logiques à l'œuvre dans la production d'alliances qui peuvent parfois emprunter à d'autres registres (coopération, entraide, migration). À cet égard, l'étude des alliances à plaisanterie est un site privilégié pour analyser le processus de recomposition des représentations et des stéréotypes, notamment autour des figures historiques de l'esclavage, de la conversion religieuse, de la rivalité, de la propriété, du pouvoir, de l'installation et de la conquête, puisque les éléments topiques de ces "plaisanteries " sont sans conteste l'esclavage, la «barbarie », la religiosité, la préséance, la gourmandise... Figures euphémisées et 
métaphoriques que donnent à voir ces joutes orales actuelles, mais figures ô combien historiques et récurrentes en Afrique de l'Ouest. Comme le rappelait Claude Meillassoux (1978: 132),

«Les brassages sociaux consécutifs aux déplacements des captifs, à la déportation des populations, aux déplacements des soldats, à la fuite des populations harcelées, aux mouvements des marchands : la menace constante qui pesait sur tous d'être capturés en même temps que le désir de chacun de jouir de la servitude des autres, ont contribué à la constitution d'un ensemble social très imbriqué, s'étendant sur des milliers de kilomètres et dont les composantes, clans, castes et classes se reconnaissent, s'opposent et s'unissent de proche en proche sur d'immenses étendues. Entre elles et les unes contre les autres, se nouent des alliances nombreuses, diverses, compulsives souvent, qui constituent par leur entrelacs un tissu social symplectique, support d'un ensemble social original dont les particularismes ethniques tendent à disparaitre au profit d'une aire de socialisation diffuse qui pénètre jusqu'au cœur de chaque État, de chaque clan $»^{29}$.

S'il faut souligner la diversité des récits d'origine de ces alliances à plaisanterie, ce sont généralement des variations sur un même motif qui empruntent aux mythes et langages politiques communs à toute la sous-région (séparation brutale, ingestion asymétrique, migration commune, incendie qui crée confusion entre deux nourrissons, inversion des rôles, échange d'attributs, tabou brisé, conquête euphémisée). Ces récits sont analogues aux mythes d'origine des groupes socioprofessionnels et des relations dichotomiques noble/griot, noble/personne de caste, aînés/cadets, oncle maternel/neveu utérin, enfants de l'oncle maternel/enfants de la tante paternelle, maître/captif, vainqueur/vaincu... À l'instar des travaux sur la production des hiérarchies sociales et des groupes professionnels en milieu malinké et avoisinant, mettant en évidence le rôle de l'alliance hiérarchique comme modèle de la relation politique et de l'intégration des étrangers (Amselle 1977, 1990, 1996 ; Murphy \& Bledshoe 1987; van Hoven 1997; Bellagamba 2002 : 77-114 ; Conrad \& Frank 1995 ; Jansen \& Zobel 1996 ; Tamari 1997 ; Giesing 2000 ; Timmer 2000 ; Schaffer 2003), l'historicisation des alliances à plaisanterie et de leurs récits d'origine permet donc de saisir la production de couples d'opposition abstraite structurants (maître/captif, patron/client, noble/personne de caste, vainqueur/vaincu, civilisé/barbare, islamisé/païen, citadin/broussard, etc.) ${ }^{30}$. Cependant, malgré l'imposition des lectures produites par les groupes dominants, les usages concrets de ces couples d'oppositions disponibles laissent une grande marge d'interprétation et de contestation pour les protagonistes, car renvoyant à un passé, sinon oublié, du moins suffisamment lointain pour être l'objet d'euphémisations et de lectures contradictoires. Ces «débats» historiques émiques, réactivés quotidiennement par les interactions d'alliances à plaisanterie, permettent l'entretien d'ethnocentrismes réciproques et la discussion publique de mémoires opposées mais partagées ${ }^{31}$. Il faut donc tenter de saisir dans l'analyse des alliances à plaisanterie ces matrices d'identifications et de relations de pouvoir qui assurent la mise en scène des relations de sujétion et de coexistence, ainsi que la production de stéréotypes marquant des frontières symboliques entre groupes (Barth 1969, Armstrong 1982). À cet égard, il est clair que la mise en frontières par la métaphore servile, la plus souvent utilisée, mériterait d'être interrogée ${ }^{32}$.

Les alliances à plaisanterie sont ainsi un des modes, parmi d'autres, de relations possibles avec «les autres» et de construction de l'extériorité (Amselle 1996) dont Claude Fay (1995, 1997), poursuivant ses analyses précédentes, montre dans ce numéro qu'elles forment un continuum (exclusion, élimination, hiérarchisation, juxtaposition) et qu'elles ont pour enjeu la propriété et le pouvoir. Au sein de cette palette de relations du « tissu 
pactuel » au Maasina, les relations de hoolaare (pacte de confiance formalisé entre groupes ethniques différents) et celles de cousinage proprement dit (dendiraagu) sont réinterprétées et manipulées à la faveur de la reformulation globale des rapports de pouvoir et de propriété de l'État malien, notamment dans le cadre de la décentralisation. La logique pactuelle entre groupes est performative, souligne Claude Fay, en ce qu'elle «fabrique " des identités contraposées, généralement associées à des propriétés professionnelles ou statutaires dont la distribution reflète les rapports de force entre ces groupes. La réciprocité des prestations n'est donc pas exclusive de la hiérarchisation des statuts. Entre contrat social, pacte politique et division du travail, l'alliance à plaisanterie constitue une articulation et permet une distribution des différences qui vise l'intégration hiérarchique et l'incorporation des dominés à travers une idéologie politique productrice d'homologies et de correspondances («Nous sommes les mêmes", "Nous ne faisons qu'un ») si fréquente en Afrique.

Dans sa contribution, Youssouf Diallo analyse les interactions de groupes peuls qui, comme les Ngoni de Tanzanie ou à l'instar des groupes commerçants, guerriers ou maraboutiques de l'Afrique de l'Ouest, font partie de ces groupes mobiles qui ont le plus grand nombre d'«alliés à plaisanterie ». Cette étude des pasteurs peuls dans l'espace interstitiel (Kopytoff 1987) de l'ouest du Burkina Faso suppose d'interroger leurs relations historiques aux royaumes de Ségou et de Kong, centres politiques dominants au XVIII ${ }^{\mathrm{e}}$ siècle, c'est-à-dire les alliances et équivalences qui se sont nouées entre ces groupes périphériques et les puissances politiques dominantes. L'anthropologie historique de Claude Fay et de Youssouf Diallo rappelle aussi comment ces alliances à plaisanterie, instituées, sont des marqueurs de relations de pouvoirs entre "gens du pouvoir ", d'une part, et « gens de la terre », du «trou » ou de la " grotte », d'autre part. Elles déclinent à la fois la répartition du pouvoir, la fabrique des identités et l'articulation des propriétés entre Dogon et Bozo, Bozo et Peuls, Peuls et Bobo... Par-delà cette production et reproduction des hiérarchies, les logiques de solidarité ne sont pas absentes de ces relations d'alliances à plaisanterie qui comportent des avantages pratiques (logement, échange de prestations, entraide cérémonielle etc.). Les alliances à plaisanterie, tout comme la convertibilité des labels patronymiques, fonctionnent comme «structure d'accueil » des étrangers (Amselle 1977, 1996), permettant l'incorporation aux groupes dominants et le rappel des différentiels de prestige et de pouvoir, comme le souligne Youssouf Diallo à travers l'exemple de la diffusion du jamu ouattara chez les groupes dominés.

Le lien entre pratiques de plaisanterie et différence des statuts avait déjà été bien mis en évidence par Robert Launay (1977) dans son article Joking Slavery. Dans ce volume, Robert Launay revient sur cette question mais sous l'angle d'une micro-politique des relations à plaisanterie. À partir d'une série de cas d'étude concernant les Jula du nord de la Côted'Ivoire, l'auteur dissocie la pratique des senankuya de toute systématisation en termes de relations humaines ou de nom (jamu) pour lui redonner son caractère purement incidentiel et interactionnel. Apparaissant entre des groupes ou des individus, cette pratique advient non pas comme un épiphénomène d'autres relations déjà instituées (en aval), mais est, au contraire, créatrice (en amont) de nouvelles relations. Ce changement de point de vue est justifié par l'observation approfondie d'une multitude de transformations identitaires, de jeux sur le choix même des cousins, de mises en cause des valeurs soi-disant instituées des cousinages, des négociations sociales ou familiales consécutives des pratiques de senankuya. Aucune de ces relations à plaisanterie n'est donc 
automatique, attendue, prédéterminée. Au contraire, elles varient pour Robert Launay selon des facteurs contextuels, loin de tout échafaudage structurel, normatif ou symbolique. Si l'ambivalence des relations de pouvoir entre les individus est mise en scène par les actes de plaisanterie, elle semble bien plus être créée par l'acte lui-même qu'en être le reflet. "Plaisanter» s'impose pour l'auteur comme une des modalités possibles d'interaction sociale, dans une situation particulière, en fonction de stratégies singulières. Plus encore, elle constitue un moyen puissant de subversion des relations hiérarchiques, des agressions ou des impositions sociales. Seule une étude pragmatique des identités et des statuts en jeu dans l'interaction semble être à même d'éviter les travers fonctionnalistes focalisant trop souvent les analyses sur les propriétés sociales implicites des senankuya, au lieu de considérer ces dernières comme des processus actifs de construction et de déconstruction des relations sociales ${ }^{33}$.

L'anthropologie politique des alliances à plaisanterie souligne ainsi à quel point ces relations font partie d'un continuum de relations sociales, dont il serait hasardeux de les abstraire. On remarque cependant que ces relations d'alliances à plaisanterie tendent à devenir le modèle sur lequel se construisent la plupart des relations d'extériorité et acquièrent de la sorte une plus grande visibilité pour le chercheur. De fait, à l'inverse d'autres pratiques de coopération, d'articulation des rapports de pouvoir, de pratiques d'hospitalité et de patronage (jatigi), ou d'extension de la parenté (mariages), les alliances à plaisanterie, en raison de leur théorisation précoce et du retour de leur visibilité comme concept, prennent, notamment dans la science politique américaine, l'épaisseur d'« institutions » et de "variable d'explication» dont les auteurs cherchent à mesurer l'éventuel rôle dans la régulation des conflits.

Conflit et coopération :

à la recherche d'une efficacité des alliances à plaisanterie?

38 Mark Davidheiser, représentant l'approche en termes de conflict resolution, analyse les enjeux de pacification autour de ces alliances à plaisanterie à partir d'une étude menée dans plusieurs villages malinké du sud-ouest de la Gambie. Soulignant combien l'approche anthropologique classique d'obédience fonctionnaliste, qui insiste sur les fonctions supposées de maintien de l'harmonie sociale jouées par ces alliances, est dépassée, il refuse cependant de minimiser le rôle et l'importance des idéologies d'harmonie sociale véhiculées par des figures de la médiation et que permettent les liens de cousinages à plaisanterie. Mark Davidheiser souligne que les médiateurs réinventent constamment des parentés fictives, des liens entre les parties en conflit en se servant du langage des alliances à plaisanterie pour tenter de résoudre des conflits qui n'ont pas été réglés par d'autres moyens. Ces alliances à plaisanterie deviennent l'un des moyens privilégiés pour créer ce lien en actualisant un script familier, perçu comme légitime et commode pour l'interaction, la coopération et les obligations mutuelles. Ce recours aux alliances permettrait de créer un espace liminal provoquant parfois des changements d'attitudes en rapprochant ces cousins médiateurs des figures classiques (et ambivalentes) de la médiation comme celle des nyamakalaw par exemple (Camara 1969; McNaughton 1988; Conrad \& Frank 1995; Hoffman 2000), des chefs religieux ou des princes déchus «faiseurs de paix » (Bazin 1988). Ces alliances doivent donc s'envisager comme des pratiques ritualisées mais malléables, les narrations invoquées amalgamant souvent des discours islamiques et assurant l'extension des sanawuya à de nouvelles situations, en fonction des contextes. 

ou non de ces cousinages envisagés comme institutions transethniques informelles et syncrétiques. À partir d'une étude de terrain dans plusieurs villages en pays serer au Sénégal, l'auteur propose de cerner les conditions de possibilité des effets des cousinages à plaisanterie en termes de promotion de la tolérance et de la coopération, et du rôle qu'ils peuvent jouer dans l'entretien d'un sentiment national. Les économies politiques locales du sens, les versions concurrentes de l'histoire des groupes sociaux, les « ressources culturelles» mobilisées par les acteurs, les intérêts politiques, le contexte d'urbanisation et de migration sont ainsi des facteurs à prendre en compte pour pouvoir mesurer au niveau local les chances de succès d'activation de rhétoriques et pratiques du cousinage à plaisanterie. Au niveau national, l'entreprise de promotion de l'unité nationale par le discours de célébration du cousinage entrepris par l'ancien gouverneur Saliou Sambou par exemple, n'est possible qu'en raison des autres adjuvants symboliques de l'unité nationale au sénégal: l'héritage populaire du diopisme, l'État culturel senghorien, l'islam confrérique, le mbalax entre autres. Par cette approche en termes d'institution syncrétique reconnaissant la part de créativité et de recombinaisons permanentes opérées par les individus, Dennis Galvan propose ainsi d'éviter les dichotomies paralysantes (le néo-primordialisme et sa critique, le néo-fonctionnalisme et sa critique) au profit d'une analyse des conditions de possibilité, tant pratiques que symboliques, des cousinages.

Des pratiques diverses

La plupart des auteurs de ce volume soulignent ainsi la large palette d'usages de ces relations, et des inventions à diverses fins (obtenir des renseignements, des faveurs, résoudre un conflit, bénéficier d'hospitalité et d'attention, sauver sa vie, faire des critiques voilées, obtenir un soutien électoral) qui font partie d'un répertoire d'actions, et qui sont actualisées en fonction de stratégies d'acteurs et de contextes de possibilité.

41 Mais les attentes de solidarité ou de pacification sont souvent contredites par les intérêts politiques et économiques en jeu. Ainsi, Dennis Galvan souligne que la politique des factions du parti dominant neutralise les effets potentiels de coopération des cousinages. Les conflits fonciers et la détérioration des conditions socio-économiques des protagonistes entravent durablement les logiques de coopération et durcissent les stéréotypes (O’Bannon 2003). Sten Hagberg rappelle que lors des conflits politiques les plus intenses au Burkina Faso, la parenté à plaisanterie n'a pas été utilisée. Ferdinand De Jong (2005) montre que la rhétorique des cousinages déployée par certains acteurs dans le conflit casamançais ne permet pas de s'attaquer aux causes réelles du conflit.

Comme le montrent tous les auteurs, les instrumentalisations diverses des relations de cousinage ne réussissent qu'en fonction des contextes de plausibilité et de légitimité. La réussite dépend de l'articulation à des intérêts, notamment des groupes dominants ou perçus comme légitimes, qui tentent ainsi d'imposer leur version de l'histoire et de la mémoire des relations entre les deux parties, leur version de l'alliance. Mais elle reste conditionnée par la ratification - ou non - par les destinataires de cette lecture, de l'idéologie produite, de la version historique mobilisée, du stéréotype proposé.

43 Les relations de pouvoir et l'asymétrie sont donc au cœur de cette micro-politique des relations à plaisanterie. Mais les contestations sont toujours possibles dans le cadre de cette relation de pouvoir. Ceci est permis notamment par la métaphore de l'esclavage et de la captivité qui est facilement retournée, comme base pour la réclamation ludique de « droits » pour le dominé du jour, et génératrice de « devoirs » pour le dominant du jour.

Cahiers d'études africaines, 184 | 2006 
Il faut en effet évoquer l'ambiguïté fondamentale de certaines interactions observées entre alliés à plaisanterie, qui permettent selon des mécanismes bien connus, l'inversion parodique et temporaire d'un rapport de domination, une critique subtile des hiérarchies, l'expression d'un message subliminal dans l'espace «tiers » que permet la plaisanterie ritualisée ${ }^{34}$. Certaines contributions de ce volume (Habgerg, Douyon) soulignent bien les ambiguïtés de la critique opérée par le biais de l'alliance à plaisanterie. Il faudrait effectivement analyser les possibilités qu'offrent les interactions d'alliance à plaisanterie de faire " passer des messages » à l'interlocuteur sans avoir l'air d'y toucher, de critiquer sans heurter de front, tout autant que la réaffirmation de stéréotypes et de préséances.

Les contributions divergent quant à l'importance à donner à la part de l'agency face à celle de la domination. Il nous semble préférable d'éviter d'enfermer le débat autour de ces questions - domination/résistance ou relation symétrique/asymétrique - afin de focaliser les observations sur l'hétérogénéité des interactions possibles, qu'il sera toujours préférable d'interpréter au cas par cas, et non selon une théorie générale et exclusive.

Une même prudence doit également conduire à se méfier de la fausse alternative entre « déclin » et « extension » de ces pratiques.

Transformations

Les transformations de ces pratiques (perçues comme en déclin pour certaines, en extension pour d'autres) à l'époque coloniale sous l'impact de l'urbanisation croissante (quoique toute relative), n'avaient pas échappé aux premiers analystes. Ainsi R.-E. Moreau (1944: 398) affirmait que ces relations étaient de plus en plus frappées d'obsolescence, en raison de la multiplication des obligations de solidarité (par la mise en contact de plus en plus fréquente de populations) difficilement supportables dans un contexte de migrations accrues et d'occidentalisation des élites ${ }^{35}$. À l'inverse, Clyde Mitchell (1956b: 35) soutenait que ces relations étaient en fait aussi récentes et «modernes», suscitées par les interactions croissantes entre jeunes travailleurs et migrants de provenances différentes dans les centres urbains et qui trouvaient là l'occasion de renouveler les plaisanteries entre groupes, symptomatiques $\mathrm{du}$ « tribalism in town $»^{36}$. James Boyd Christensen (1963: 1325) soutenait également que les relations d' utani en Tanzanie étaient en voie d'extension et non de contraction en raison de l'introduction du concept de «tribu » par les Européens qui a permis la standardisation et la diffusion de ces relations sur des espaces plus larges. Autrement dit, que ce soit à l'époque précoloniale (Amselle 1977; Perinbam 1997; Fay 1995, 1997), coloniale ou postcoloniale, il faut considérer ces relations à plaisanterie comme sujettes à des processus permanents de création, de renforcement ou d'obsolescence.

47 En ce qui concerne le temps présent, on observe un certain tarissement des obligations, solidarités ou facilités attendues de ces relations (qui fait d'ailleurs l'objet de déplorations chez certains protagonistes, ou à un niveau plus général, par les intellectuels engagés dans leur promotion). Au Maasina, Claude Fay souligne un "assèchement des pactes " dont l'enjeu n'est plus sensible: ne se maintiennent que ceux qui sont utiles pour les individus, tandis qu'on assiste à une euphémisation des autres. Dans le contexte de démocratisation et de décentralisation, si les entrepreneurs politiques tentent certes de manipuler ces logiques pactuelles réifiées, l'efficacité semble très mitigée. Dennis Galvan rapporte également qu'en ville, la paupérisation et le conflit pour les ressources peuvent remettre en cause des liens de " cousinage " pratiqués au village. Même en milieu rural, ces «cousinages » sont parfois davantage une évocation du passé qu'une pratique du présent dans un contexte de raréfaction des ressources (O’Bannon 2003). 
Cependant, les contributions de Denis Douyon et Cécile Canut soulignent que dans le contexte urbain, où la convertibilité des identités permise par l'anonymat est encore accrue, offrant ainsi des possibilités de dissimulations, de conversions, de changements de statuts, les occasions d'activation de parentés fictives, sur le modèle des relations à plaisanterie, se multiplient. Elles s'étendent à des individus et des groupes qui n'étaient pas préalablement insérés dans ces réseaux de relation, que ce soit par le biais des patronymes alliés dont le nombre tend à s'étendre ou par celui de la standardisation des relations entre groupes, souvent par transitivité. Par ailleurs, comme l'évoque Mark Davidheiser, les jeunes ont tendance à se «traditionaliser» en vieillissant, et de nombreux interlocuteurs admettent de fait découvrir «l'utilité » de ces relations avec l'âge.

49 L'instrumentalisation des relations à plaisanterie est le plus sûr indicateur de la vitalité de ces pratiques pour lesquelles il se trouve constamment des occasions d'actualisation, car les acteurs en attendent certains bénéfices, comme le montre Denis Douyon lorsqu'il analyse la «senankuyamania» dans la vie politique malienne. L'évidence de ces transformations et de ces recompositions permanentes de la logique des pactes et alliances conduit donc à proposer une analyse aux antipodes de toute idée de perpétuation à l'identique de supposées «pratiques ancestrales ». C'est sans doute dans ce domaine des usages stratégiques et d'instrumentalisations que les recherches les plus prometteuses restent à faire ${ }^{37}$.

50 Au-delà de ces pratiques concrètes, le présent volume se propose donc de rendre compte du déploiement des alliances à plaisanterie (pratiques et récits) dans l'espace public et médiatique contemporain à des fins ludiques mais aussi stratégiques. Les pratiques de plaisanterie entre certains groupes, caractérisant des "alliances», redéploient dans l'espace médiatique les questions de préséance qui se jouent symboliquement.

Espaces publics et performance

51 Les espaces privilégiés de déploiement renouvelés de ces pratiques sont bien sûr les espaces publics et les scènes politiques contemporaines, à destination d'un "public »: lecteur de journal, auditeur de radio en langue nationale, spectateur, électeur.

La pratique peut être essentiellement ludique, ainsi que l'illustrent de nombreux exemples issus de la chanson contemporaine. Ainsi, par exemple, la présentation, par les humoristes «chanteurs » sénégalais Tonton Ada et Sylla Mounial, d'une lutte entre un Serer et un Toucouleur visant à les départager une fois pour toutes (Étienne Smith dans ce volume), ou bien la chanson Sordassi kè du chanteur guinéen Cheikh Pénor Traoré moquant publiquement la gloutonnerie des Condés8, ou encore celle de la chanteuse guinéenne Mama Diabaté ${ }^{39}$.

Certains journaux jouent un rôle prépondérant dans la mise en écrit et en dessin des relations à plaisanterie, de façon quasi systématique dans le cas du Journal du Jeudi au Burkina Faso (Sten Hagberg dans ce volume qui analyse la ligne éditoriale de ce journal dirigé par Boubacar Diallo), mais aussi dans le journal guinéen Le lynx, ou bien l'observateur Paalga au Burkina Faso (« Nobila-cabaret» alias Boniface Batiana) ou dans les journaux sénégalais L'Observateur ou Le Quotidien (rubrique d'été Le Coin du Couz' dans laquelle le journaliste Soro Diop ridiculise les Ndiayène). Certains politiciens, certaines personnalités ou des citoyens ordinaires y sont l'objet de remarques ironiques, de la part de journalistes attentifs au moindre « saalit » de leurs cousins, si haut placés soient-ils. 

certaines codifications pour s'intégrer dans le champ de l'«humour ethnique » universellement pratiqué (Davies 1990; Gundelach 2000 ; Vucetic 2004) et emprunte au registre mondialisé des plaisanteries. Le contenu des échanges de plaisanteries est parfois influencé par les motifs globaux d'histoires drôles qui circulent notamment par le biais d'Internet. Ainsi cette "Lettre d'une maman toucouleur à son fils » dans le journal sénégalais Le Quotidien, qui se présente comme une raillerie typique (sous-entendu de la part des Serer) envers les Toucouleur est l'exacte reprise d'une blague sur les blondes qui circule en Europe $^{46}$. De même, sur certains forums Internet, les blagues émises empruntent tantôt au registre habituel des alliances à plaisanterie (gloutonnerie, esclave/ roi, type physique), tantôt au registre mondialisé des blagues. Bien que ces alliances à plaisanterie conservent en partie leur spécificité - car malgré les extensions soulignées, cet humour ne cible pas n'importe quel groupe, mais bien ceux concernés par ces alliances - n'assiste-t-on pas en effet à une évolution vers des pratiques plus classiques de « railleries ethniques » qui tendent de plus en plus à devenir un sous-genre particulier de cet humour ethnique universel. Quoi qu'il en soit, ces usages médiatiques soulignent bien la créativité et la plasticité de ces pratiques, que l'on ne peut réduire à de simples instrumentalisations.

Les comiques ne sont pas en reste comme par exemple Baïlo Sow, en Guinée, se moquant des Diakhanké (Kouyaté 2003 : 32). Au-delà des radios rurales et communautaires qui sont parfois le lieu d'expression de ces pratiques de plaisanterie. Ainsi, les émissions Rions un peu et Éclats de rire au Mali, par exemple, qui offraient une large place aux appels Burkina qui raille par imitation des accents ou des accoutrements les «types » peuls ou bobo (Sissao 2002: 152). Le théâtre populaire emprunte aussi largement à cette thématique. décor culturel de l'intrigue (Kourouma 1990 : 37, 42, 117, 183 ; Ba 1991 : 266s, 275s, 316, 1994 : 91, 103, 180; Ndiaye 1999: 13-14; Prignitz 2001; Sissao 2002) ${ }^{41}$ ou qu'elles constituent un des moyens de la narration comme dans Peuls de Tierno Monenembo (2004) qui s'ouvre sur cette dédicace : « Pour ces idiots de Sérères $»^{42} \ldots$

de cousinage. Il s'agit le plus souvent d'une pratique ludique entre internautes, mais aussi parfois d'un débat sur ces pratiques, leurs sens et leur avenir ${ }^{43}$.

emprunte largement dans le stock des stéréotypes ethniques disponibles, et à l'esprit des alliances à plaisanterie, sans s'y limiter. L'ethnicité, sous cette forme ludique, est omniprésente dans l'espace public des pays concernés. La dimension ludique, pour essentielle qu'elle soit, ne doit cependant pas faire oublier l'ambiguïté du cadre de cette expression médiatisée de stéréotypes ethniques, pas toujours réciproques ${ }^{45}$.

Toutefois, ces instrumentalisations, notamment dans le monde politique, existent bel et bien. Ces pratiques d'alliances à plaisanterie peuvent faire le miel des hommes politiques, même si leur utilisation dépend des contextes, du rapport à la culture populaire et au "style politique» propre de chaque homme politique. Ainsi le président Senghor au Sénégal ou Lamizana au Burkina Faso ont fait de la rhétorique du cousinage une 
constante de leur pratique politique vernaculaire. Les hommes politiques maliens, comme le montre Denis Douyon dans son analyse des usages et mésusages des senankuya dans la vie politique malienne, tendraient même à l'utiliser de plus en plus. Ces usages stratégiques, souligne-t-il, donnent lieu à toutes sortes de manipulations : «le cousinage est devenu le moyen d'énoncer les grands principes et de faire de petits arrangements. » Mais la part de stratégie et de ruse n'est pas l'apanage des élites politiques, car les individus-électeurs savent également l'utiliser pour tenter de faire entrer l'élu dans une relation morale d'accountability et reformuler les logiques clientélistes à leur avantage. Les contributions de Sten Hagberg et Denis Douyon invitent donc à penser la part de ces pratiques d'alliances à plaisanterie dans la formation d'une culture politique burkinabè ou malienne. Les alliances à plaisanterie font partie d'un savoir culturel partagé entre élites politiques et populations comme le rappelle Sten Hagberg, reprenant l'analyse d'Achille Mbembe (2000) sur la communauté épistémique commune aux gouvernants et gouvernés en postcolonie qui relève à la fois du partage d'une bouffonnerie ritualisée et d'une domination d'autant plus hégémonique. Cette ambiguïté se retrouve dans les représentations sur les qualités morales qui font un «bon politicien ». L'imputation de la pratique du cousinage à un leader politique est tantôt positive (« il pratiquait bien », « il pratiquait beaucoup », «il restait enraciné dans sa culture », « il était plus proche des gens ») ${ }^{47}$ tantôt négative («il en usait et en abusait », «il peut tromper les gens », « il jouait trop de la corde sensible »). Au-delà de cette ambivalence constitutive, les alliances à plaisanterie sont donc essentielles à la compréhension des imaginaires politiques. Comme l'affirme Sten Hagberg, « la parenté à plaisanterie ouvre un espace culturellement construit pour les rires, satires et amusements dans la culture politique burkinabè ».

Au-delà du repérage d'une culture politique partagée, il faut analyser comment ces pratiques s'exercent dans les lieux de pouvoir, dans l'enceinte des assemblées, des administrations, des entreprises, dans le commandement territorial ${ }^{48}$, lors de meetings et tournées politiques, des grands événements nationaux ${ }^{49}$, ce à quoi s'attellent certaines études (Canut, Davidheiser, Douyon, Fay, Galvan, Hagberg, Smith). Toutefois, en raison des visions romancées données par les acteurs de l'utilisation du cousinage à plaisanterie dans le fonctionnement politique quotidien des institutions, la prudence est nécessaire en ce domaine et les analyses doivent toujours se fonder sur l'observation. Ces études s'intègrent enfin dans la problématique plus large de l'utilisation du langage et de la métaphore de la parenté en politique qui, loin d'être spécifique à l'Afrique et aux alliances à plaisanterie, prend dans le cadre de ces alliances une dimension encore plus évidente.

61 Mais au-delà de ces pratiques ludiques et stratégiques individuelles, les alliances à plaisanterie sont également prises dans des logiques discursives de patrimonialisation qui s'inscrivent parfois dans le cadre de véritables politiques symboliques d'État.

Savoirs locaux, audience globale :

canonisation de la tradition, acte 2

«Le sinangouya ou "cousinage à plaisanteries" est l'un des traits saillants de la culture malienne et le socle de l'unité nationale. Véritable institution précédant toutes les autres, il permet à deux, voire plusieurs, ethnies, races ou communautés de se tourner en dérision, mais aussi - et c'est là le plus important - de pacifier les rapports sociaux grâce à l'intercession en cas de conflit notamment [...].

Des informations complémentaires sur le "cousinage à plaisanteries" seraient appréciées $»^{50}$. mise en discours des alliances à plaisanterie à des fins de promotion, processus dont 
l'actualité n'est plus à découvrir. La « production en masse des traditions » (Hobsbawm \& Ranger 1983 ; Anderson 1983 ; Thiesse 1999 ; Dimitrijevic 2004), constat devenu classique, trouve ici un vaste champ d'analyse. Ce large mouvement de standardisation des identités, de canonisation des mythes et traditions orales et de « recyclage de la tradition anthropologique " (Hagberg dans ce volume), observable en Afrique comme ailleurs, place dans les États considérés ici ces relations à plaisanterie au cœur du processus. Ce phénomène d'ampleur, à propos duquel nous manquons sans aucun doute de recul, donne lieu à des interprétations divergentes, y compris au sein des différentes contributions rassemblées ici.

Au-delà des conflits d'interprétations, trois types de processus sont toutefois régulièrement mis en lumière dans l'ensemble des travaux: stratégies de patrimonialisation, essais de créations de communautés nationales imaginées, et construction de discours à destination du reste du monde. Bien qu'ils soient intrinsèquement liés, nous proposons de les décliner séparément.

Logiques de patrimonialisation

La patrimonialisation des alliances à plaisanterie, considérées comme faisant partie d'un héritage culturel d'ensemble à préserver, est, malgré des objectifs souvent divergents, le produit conjoint de créateurs, journalistes, entrepreneurs culturels, associations, chercheurs ou hommes politiques. Cette stratégie de patrimonialisation transforme la pratique, à laquelle se greffe désormais un discours réflexif de valorisation et d'explication de cette pratique à destination d'un public.

La chanson contemporaine, formidable vitrine pour un pays comme le Mali par exemple, s'empare ainsi des senankuya dans un souci de valorisation du pays et de ses "valeurs " supposées uniques : la chanteuse Rokia Traoré51, ses compatriotes Habib Koïte (chanson Baro) et Adama Yalomba (chanson Jamana), ou encore le chanteur guinéen Pathé Moloko (chanson Sanakuyagaal) $^{52}$, la chanteuse sénégalaise Ndèye Kassé (chanson Serer) ${ }^{53}$, ou le groupe nigérien Sogha (chanson Baaseterey) actualisent par exemple cette thématique patrimoniale. Le réseau des radios rurales et communautaires, autre vecteur de la médiatisation patrimoniale, joue un rôle actif dans le recueil et l'activation sur les ondes des légendes sur l'origine de ces cousinages, associées à des récits sur les fondations de village. Les différentes versions sont parfois débattues en direct entre les invités et les auditeurs. Se constituent de la sorte de nouveaux corpus de «traditions » des cousinages. Au-delà des radios, toute une série d'acteurs, des ministères concernés aux ONG, sont engagés dans la patrimonialisation de cette "pratique ", sorte de "mapping " $^{54}$, qui fait désormais partie de la politique culturelle des États, en partenariat avec des institutions relevant de l'Union africaine comme le CELHTO de Niamey ${ }^{55}$ et des acteurs internationaux comme Intermédia consultants, le Club du Sahel, ou Enda Tiers-Monde. Ainsi, au Mali, on peut relever l'entrée des "parentés à plaisanterie» au musée national ${ }^{56}$. L'audiovisuel s'empare en effet de cette thématique. À partir d'un séjour au Burkina et au Sénégal, le réalisateur belge Jacques Faton (1999) a publié un livre et réalisé un film documentaire sur le sujet ${ }^{57}$. Au Burkina Faso, le directeur du Journal du Jeudi, Boubacar Diallo, a également réalisé un film de promotion de la parenté à plaisanterie (Hagberg dans ce volume).

La prise en charge étatique du discours de promotion des alliances à plaisanterie s'est amplifiée ces dernières années comme en témoignent la Semaine nationale de la culture au Burkina Faso et les Grandes conférences du ministère de la Culture, qui ont largement traité du thème (Kompaoré 1999 ; Nyamba 1999 ; Topan 1999), ainsi que les Festivals des 
origines et autres Journées culturelles au Sénégal, donnant lieu à des colloques et publications sur le sujet (Tambadou 1996; Sambou 2005), ou encore les manifestations culturelles de l'État malien, comme la Biennale des Arts ou les festivités célébrant la Charte de Kurukan Fuga ${ }^{58}$. Ainsi, l'année 2003 au Mali a-t-elle été décrétée année de promotion des senankuya. Dans plusieurs manifestations culturelles de l'État burkinabè, des plages horaires réservées à la parenté à plaisanterie sont aménagées dans le programme officiel ${ }^{59}$. On note l'organisation de matches de foot entre Bisa et Gurunsi, San et Moose et dont l'enjeu est de perdre, afin de ne pas gagner le trophée infamant " confirmant » le stéréotype (tête de chien, panier d'arachides, etc.). Lors de la Semaine nationale de la culture, est installé un "village des parents à plaisanterie » ou «village des communautés » fonctionnant sur l'émulation " patriotique », festive et culinaire des différentes communautés représentées (Sissao 2002 : 152,155), comme lors des Festivals des origines Serer-Joola au Sénégal. Toutes ces manifestations concourent à l'institutionnalisation des cousinages et à leur disciplinarisation par les grands rituels étatiques.

La problématique des alliances à plaisanterie fait également l'objet de financements officiels. Au Burkina Faso, par exemple, au-delà des Grandes Conférences, le thème des alliances à plaisanterie est inscrit comme thème prioritaire de recherche par le Centre national de la recherche scientifique et technique (CNRST 1995) et a donné lieu à plusieurs enquêtes de terrain (Sissao 2002). Selon le document du CNRST (1995: 360), « il paraît opportun de les [les parentés ou alliances à plaisanterie] identifier, de les classer et de les analyser afin d'en tirer un meilleur parti ». Au Sénégal, le rapport du colloque sur les Convergences culturelles de 1994 préconise de même la réalisation d'études sur "l'impact de la parenté à plaisanterie » (Tambadou 1996: 36). Différents ateliers sous-régionaux (Labbé 1997, Bamako 1997, Kankan 1998, Mopti 1999, Bamako 2004, Conakry 2005) organisés par l'ong Enda Tiers-Monde, la coopération suisse ou bien le Club du Sahel ont également établi un programme de collecte d'informations sur la "parenté plaisante » dans toute la sous-région, sous forme écrite (Camara 1969; Diall \& Traoré 1999; Teme 1999 ; Thea 1999) et sous forme d'émissions pour les radios communautaires (archivées au CELTHO de Niamey, projet ARTO).

Dans ces entreprises de promotion, on trouve quantité d'acteurs aux statuts et objectifs différents. Des individus qui activent le registre de la légitimité «traditionnelle», mais qui sont de fait bien plus que des traditionnistes ou responsables politiques «traditionnels», comme El Hadj Sékou Tall (1996), le Larlé Naaba Tigré, qui dirige la revue Tradition et Modernité au Burkina Faso ${ }^{60}$, Bakary Soumano, ancien « chef des griots » au Mali ${ }^{61}$. Mais aussi des chercheurs comme Raphaël Ndiaye de Enda Tiers-Monde ou Alain Sissao, des journalistes comme Martin Faye ou Boniface Batiana, des animateurs d'émissions culturelles à la radio ou à la télévision, des hommes de théâtre comme Prosper Kompaoré, des écrivains comme Cheikh Hamidou Kane, des érudits comme Saliou Kandji, des militants des langues nationales comme Babacar Sédikh Diouf ou Yoro Doro Diallo, un gouverneur comme Saliou Sambou au Sénégal, un magistrat comme Siriman Kouyaté en Guinée, Mgr Anselme Sanon au Burkina, le directeur du CELHTo Mangoné Niang à Niamey. Certains de ces entrepreneurs culturels fournissent une expertise sur le sujet des « alliances à plaisanterie » et sont consultés par divers acteurs internationaux ${ }^{62}$. Derrière un discours unanime se nichent évidemment des querelles de positionnement, des tentatives de monopolisation de l'expression du savoir local, des contestations de 
légitimité, mais aussi la volonté de valoriser et «sauver » un héritage culturel perçu comme menacé.

On remarque, à cet effet, la création d'associations de promotion comme l'Association burkinabè de promotion de la parenté à plaisanteries (AB3P) en 2001 à Ouagadougou, ou l'Association pour la promotion de la parenté à plaisanteries à Bobo-Dioulasso (Sissao 2002 : 154), de l'Association Ageen et Diambogne au Sénégal en 1994 (De Jong 2005), dont l'objectif affiché est de promouvoir et ré-inculquer à la jeunesse la "parenté à plaisanterie » et les valeurs qui y seraient associées ${ }^{63}$.

70 L'émergence de ces associations est indissociable d'un constat fait par leurs fondateurs d'une supposée crise de la transmission et d'une plainte à propos de la « perte des valeurs traditionnelles ». Comme pour les langues en danger, il conviendrait de réapprendre aux jeunes afin qu'ils n'oublient pas $^{64}$. L'inflation des discours de promotion semble corrélative du constat opéré par les fondateurs de ces associations du « déclin » supposé des pratiques. Cette tentative de mettre la « tradition » à « l'avant-garde », comme avenir possible (Diagne 1992), ce discours de la nécessité du « recours culturel » (Poncelet 1994), s'intègre tout à fait dans une stratégie de promotion de compétences et de recherches de financements (de recherches ethnologiques, des associations de promotion et de redécouverte).

71 Ces associations au Sénégal et au Burkina Faso ont notamment réclamé la création de journées nationales de la parenté à plaisanterie, d'une « journée sahélienne de la parenté à plaisanterie » dont les manifestations principales devraient se dérouler à Kurukan Fuga, d'une "semaine du cousinage", de "colonies de vacances" entre groupes alliés à plaisanterie, d'une " fête du cousinage », etc. ${ }^{65}$. Plus encore, l'enseignement des alliances à plaisanterie dans le cadre scolaire et la confection de matériel didactique sur ces alliances pour les cours d'éducation civique a été réclamé au Burkina Faso (Sissao 2002 :135), et même réalisé au Sénégal grâce à la collaboration d'oNG ${ }^{66}$. C'est là un retour sous forme écrite des « prescriptions » éthiques des liens d'alliances à plaisanterie ${ }^{67}$. Cette didactique néo-fonctionnaliste des parentés à plaisanterie, c'est-à-dire cette volonté de standardisation de ces pratiques en mécanismes de pacification et de résolution des conflits, vise à les faire passer du statut de pratiques spontanées, résultant comme le montrent les différents contributeurs de ce volume, de stratégies diverses et changeantes dans l'interaction, à celui d'« outil », pour une fin bien déterminée et constante. Tout en admettant une éventuelle efficacité des discours de pacification et les intentions louables, on ne peut que souligner l'illusion de modelage du social, de réinculcation scolastique de « valeurs » absolutisées de ce discours pédagogique. La même idéologie de «la paix par la culture » figure dans le kit des "bonnes pratiques" pour prêcher la réconciliation intercommunautaire en Côte-d'Ivoire de l'oNG américaine Search for Common Ground, qui, à la demande des radios locales partenaires, utilise ces alliances à plaisanterie dans le cadre de l'émission radiophonique Passerelle ${ }^{68}$.

Ces différents acteurs accomplissent donc un travail culturel de standardisation et de rationalisation. Dans cette optique patrimonialiste classique, les «ethnies» sont considérées comme des espaces de création, et leur diversité comme une richesse. Que cette patrimonialisation culturelle aboutisse, selon les uns, à une réification et à une dénaturation des pratiques, ou, selon les autres, à une créativité et de nouveaux usages, ce sont aussi ces mises en scène qui constituent désormais, pour les acteurs, la «tradition». Ce qui, assurément, ne manque pas de créer des malentendus entre les promoteurs de ces alliances et les chercheurs d'une part, et avec la majorité des individus 
pour qui la question de la patrimonialisation ou la «survie" de telles pratiques quotidiennes ne se pose pas, d'autre part.

La création des identités nationales

Les logiques de patrimonialisation mentionnées ci-dessus s'inscrivent dans le renouvellement des projets nationaux et nationalistes en Afrique de l'Ouest, dont on peut apprécier la continuité - ainsi que l'adaptation au nouveau Zeitgeist - avec les formulations antérieures de l'époque des indépendances. Le discours nationaliste est entré en crise, mais trouve dans la patrimonialisation des alliances à plaisanterie l'occasion de renaitre. Les ficelles classiques des manifestations du nationalisme officiel sont mises à contribution dans le discours de célébration des alliances à plaisanterie. Alors que les nationalismes des « soleils des indépendances » tendaient souvent à nier les ethnicités perçues comme concurrentes du projet d'intégration nationale, le nationalisme contemporain analysé ici se trouve pris dans le Zeitgeist mondial de la «diversité culturelle ", ce qui l'oblige à composer avec les identités culturelles des différentes communautés.

L'insistance sur les alliances à plaisanterie comme illustration (voire cause) de relations interethniques pacifiques entre les composantes de la nation sert-elle d'argument pour formuler la singularité dont chacun de ces pays se prévaut? Comme le montrent les citations en exergue, les exceptions sénégalaise, burkinabè, malienne, guinéenne, nigérienne, zambienne, etc., devraient beaucoup à ces alliances à plaisanterie qui «cimentent les liens à l'intérieur des nations " ${ }^{69}$. L' « héritage » à maintenir (Kouyaté 2003), le "trésor » à faire fructifier sont les métaphores utilisées pour décrire les dons propres à ces nations qui doivent s'en montrer «dignes" parce qu'ils les rendent « uniques ». L'image du pays promue à l'étranger fait donc la part belle à ces alliances à plaisanterie comme, par exemple, sur plusieurs sites officiels au Burkina Faso, au Mali ou au Niger. Ainsi, cette présentation officielle du Niger pour les $V^{\text {es }}$ Jeux de la francophonie de 2005: «La force de la cohésion sociale au Niger, c'est sans doute le cousinage à plaisanterie. Ce cousinage permet aux différents groupes de se critiquer entre eux sans aucun heurt. C'est un excellent moyen de résorber les problèmes interethniques et cela fait de la société nigérienne, une société tolérante, une nation de coexistence pacifique $»^{70}$ . Parfois véritable «travail culturel» à l'exportation, les discours de promotion sont portés dans des enceintes internationales fort diverses. On note ainsi la «Conférence sur la parenté à plaisanterie », organisée par le ministre burkinabè des Relations avec le parlement à l'ambassade du Burkina Faso à Paris le 25 mai 2002, la conférence sur le cousinage au Mali prévue lors des "Journées culturelles du Mali à Vienne", en octobre 2006, organisées par le ministère des Maliens de l'Extérieur dont le programme annonce : «Peut-on dire que toute l'Afrique est pleine de conflits ethniques? Toute l'Afrique ? Non, le Sahel central, surtout les pays tels que le Mali ou le Burkina Faso, sont caractérisés par une société multi-ethnique stable. Beaucoup de Maliens pensent que cette harmonie interethnique dans leur société est due à l'existence de cousinages qui permettent de se moquer des gens et de résoudre certains conflits à la source même. » Dans les conférences internationales ou les interviews, les représentants du Burkina Faso, du Mali ou du Sénégal se font fort de rappeler l'existence de cette «coutume » qui expliquerait la paix sociale dans leurs pays respectifs. Ainsi, le message de l'ambassadrice du Mali au Japon sur le site de l'ambassade fait jouer au senankuya un rôle moteur : «There is a custom in Mali known as "Sananguya", passed down through the generations in the different ethnic groups, which says that we should not dominate but support one another while laughing 
and joking, irrespective of the place or circumstances. Japanese textbooks cite the efforts of Malians to build friendly interpersonal relations as an example of a culture that places importance on greetings $»^{71}$.

Ces récits fonctionnent comme la valorisation d'une « expertise nationale $»^{72}$. Dans cette optique, promotion touristique et célébration des alliances à plaisanterie ne font souvent qu'une $^{73}$. Ce discours n'est d'ailleurs pas propre aux États considérés, car la suggestion (paradoxale ?) de l'exportation de ces « pratiques de paix », propres au " génie » national, aux autres continents qui en sont démunis, est repris par des intellectuels provenant d'horizons divers ${ }^{74}$.

Pour les acteurs locaux, la logique est d'ancrer ces formulations nationales dans le passé, leur donner une profondeur historique, processus classique de naturalisation de la communauté imaginée, visant à faire croire que les nations et le sentiment national préexistent aux États hérités de la période coloniale. Ces groupes reliés entre eux par le cousinage auraient ainsi eu la conscience « ancestrale» de leur destin commun. Selon ce récit, les alliances à plaisanterie seraient au minimum l'indice, au maximum la cause, d'un sentiment national fondé sur une unité culturelle antérieure aux États. Le discours d'Amadou Toumani Touré, clôturant la Biennale des Arts de 2003, est un bon exemple de ce processus discursif de naturalisation de la nation malienne : «Exception en Afrique, rarement un peuple a préexisté de manière aussi incontestable à un État. Cimentée par l'histoire, la conscience malienne est une réalité, fondée sur la conviction collective d'appartenir à une nation, dont l'âge et l'étendue dépassent largement ceux de l'actuelle république du Mali. Diversité ethnique et solidarités culturelles. Cet état d'esprit découle des faits : à un moment donné d'une histoire plus que millénaire, chacune des grandes ethnies a connu une période de gloire, en régnant sur les autres. Les relations entre les groupes ethniques ont été consolidées par le brassage entre les communautés et fortifiées par le sinankuya ou cousinage à plaisanteries $»^{75}$. Le récit nationaliste malien proclame ainsi que les senankuya sont des "ficelles relationnelles », héritage d'un passé de diversité et de brassages ${ }^{76}$. Elles sont l'indice visible que la " démocratie » et la "décentralisation " ne sont pas une importation mais une tradition locale, relevant d'un savoir-faire malien depuis l'Empire « fédéral » de Sunjata Keïta et la Charte de Kurukan Fuga ${ }^{77}$.

Il convient donc d'analyser comment les alliances à plaisanterie sont intégrées dans ces récits nationaux des convergences, et transformées en nœuds de connexion des différentes communautés au sein de l'ensemble national (De Jong 2005 ; Galvan et Smith dans ce numéro). Dans ce récit, les alliances à plaisanterie fonctionnent comme opérateurs symboliques du passage de la pluralité des « ethnies » à la «nation », fruit de leur combinaison. L'objectif, somme toute très renanien (Renan 1882), consiste à donner une valeur d'intégration aux conflits du passé (qu'ils soient nommés comme tels ou euphémisés en " alliances », « brassages » et " convergences ») et donc à ces alliances qui en sont la trace vernaculaire et le souvenir ludique (Smith dans ce volume).

78 À ce niveau, certaines des interprétations des contributeurs de ce volume divergent quelque peu : faut-il voir dans cette articulation la volonté de créer un sentiment national par « connexité » (Smith, Galvan) ou l'indice de replis communautaires favorisés par un discours ethnicisant (Fouéré, Canut)? La conciliation de ces deux approches reste toutefois possible à travers l'analyse de l'ambiguïté et des difficultés inhérentes au projet d'articulation des différences communautaires et de l'unité nationale. Marie-Aude Fouéré et Cécile Canut soulignent, en ce sens, qu'il s'agit avant tout d'un discours réactif. La réaction concerne d'une part la matérialité de ces conflits et, d'autre part, la lecture 
ethniciste véhiculée par le discours dominant des médias occidentaux qui contribue fortement à la construction d'un discours nationaliste dérivé. Ce discours s'est saisi de l'objet disponible "parenté à plaisanterie ", codifié et prêt à l'emploi au rayon des traditions répertoriées de la bibliothèque coloniale, afin d'apporter des « remèdes » et de canoniser un «outil» essentiel à l'intégration nationale ${ }^{78}$. Dans une Afrique présentée systématiquement comme le continent déchiré par les conflits ethniques ${ }^{79}$, l'objectif serait, de manière originale et africaine, de trouver les moyens de "recoller les morceaux» (sous-entendu ethniques), de «colmater les brèches» entre les particularismes, pour reprendre l'expression senghorienne. À cet égard, on peut remarquer l'ambiguïté intrinsèque du discours valorisant à la fois les différences communautaires (nécessaires pour que les alliances à plaisanterie fonctionnent) et les convergences dans l'unité nationale (De Jong 2005).

On notera cependant que les discours de valorisation des alliances à plaisanterie propices à l'expression, au plan externe, d'une singularité africaine, au plan interne, émergent précisément en contrepoint des logiques de l'autochtonie (Bayart, Geschiere \& Nyamnjoh 2001) que ce soit au Sénégal avec la crise casamançaise (De Jong 2005 ; Smith dans ce volume), ou au Burkina de la part d'acteurs de la société civile qui trouvent là l'occasion de critiquer la manipulation de l'ethnicité par certains politiciens (Hagberg dans ce volume). Si ces discours de célébration des alliances à plaisanterie contribuent sans doute, par leur dimension performative, à standardiser voire à durcir des identités, il est difficile d'y voir, dans l'intention, une logique communautariste, de l'ethnisme ou du localisme, puisqu'au contraire il s'agit de récupérer, domestiquer, et réordonner les récits ethniques dans le grand récit national(iste) (Smith et Galvan dans ce numéro ; De Jong 2005). Cette entreprise de promotion des cousinages s'adosse au pari que reconnaitre culturellement les ethnicités, les célébrer ensemble pour délégitimer les imaginations séparatistes, permettrait d'éviter toute utilisation politique de la différence. Selon cette prémisse, l'ethnicité devrait être culturellement légitime pour être politiquement illégitime.

Par ailleurs, ne faut-il pas distinguer intentionnalité et conséquences? On peut de la sorte souligner les instrumentalisations diverses des alliances à plaisanterie par des acteurs avec des objectifs différents, mais dont l'effet commun parfois involontaire est de contribuer à valider la catégorie " ethnie ». Ce discours est une réaction "de l'intérieur " même de cette catégorie. Ne pouvant se résoudre à l'abandon de la notion comme l'y appelle l'anthropologie déconstructionniste, tout en étant une réaction à l'ethnisme, ce discours a pour objectif affiché de sauver l'« ethnie » (passablement essentialisée, réifiée et folklorisée au passage) de l'autochtonie en investissant les relations interethniques des vertus agglutinantes et pacificatrices supposées des alliances à plaisanterie pour produire des ethnicités non closes. La catégorie " ethnie » est donc à la fois confirmée et dépassée. Pour leurs promoteurs, il s'agit de la mobilisation politique des alliances interethniques ou trans-ethniques, donc de l'entre deux des groupes, et non des « ethnies» en tant que telles, même s'il est clair que cette mobilisation conduit à des processus d'essentialisation et de réification similaires à ceux produits dans la mobilisation "ethnique $»^{80}$. Leur objectif n'en est pas moins de montrer que les « ethnies " peuvent être transcendées non seulement par la citoyenneté importée, mais aussi et surtout par la civilité trans-ethnique locale, "précoloniale», jugée par eux plus attentive à l'altérité dans l'articulation nécessaire des différences que "l'universalisme abstrait » (Smith dans ce volume). Une des voies de sortie de ce débat entre les auteurs est de le réinscrire - et de la sorte le 
banaliser - dans la problématique plus large et comparative des différents modèles d'intégration nationale en débat en Afrique, afin de sortir des faux débats "État » vs "ethnies", alternativement chargés de tous les maux, en soulignant à la fois les contextes nationaux différents des nationalismes africains et les problématiques communes (Berman, Eyoh \& Kymlicka 2004 ; Forrest 2004 ; Barrington 2006).

On note en outre que cette incorporation de la problématique des relations à plaisanterie, dans les projets nationalistes, n'est pas exclusive d'une application à l'espace sousrégional chez la plupart de ses promoteurs. L'existence de ces liens dans toute la sousrégion ouest-africaine, la transitivité des alliances, les migrations anciennes, et les équivalences patronymiques sont ainsi mises en valeurs (Mariko 1990; Ndiaye 1992; Sissao 2002; Kouyaté 2003). La référence aux espaces d'intégrations du passé, l'imagination rétrospective de Kurukan Fuga et des espaces impériaux plus vastes que les actuels États-nations, ou à cheval sur plusieurs d'entre eux (Ghana, Mali, Gabu) ${ }^{81}$, bien qu'elles restent souvent à l'état de célébrations rhétoriques, sont omniprésentes dans le discours de promotion.

Cosmopolitisme du national

Le rôle de « l'Occident » dans le processus de réinvention de traditions nationales et des logiques de patrimonialisation des relations à plaisanterie donne lieu à des interprétations divergentes entre les auteurs. Cécile Canut et Marie-Aude Fouéré soulignent à quel point les acteurs du développement ou de la coopération du nord, notamment par le biais des financements, jouent un rôle prépondérant dans cette invention néo-fonctionnaliste de traditions locales d'alliances à plaisanterie. Le rôle des acteurs extérieurs dans l'essor des inventions nationales ou régionales et des constructions identitaires est en effet fondamental et bien connu, comme l'ont montré les nombreux travaux sur les nationalismes européens des deux derniers siècles (Hobsbawm \& Ranger 1983 ; Thiesse 1999). L'on peut, à ce propos, reprendre la formule d'Anne-Marie Thiesse (ibid.: 64) de «cosmopolitisme du national». Ce recours à la dimension comparative permet précisément de relativiser non pas le poids de la «bibliothèque coloniale", mais sa spécificité. Certes l'UNEsco a remplacé les frères Grimm dans l'inventaire et la promotion des traditions "nationales", dans les "entreprises d'assistance identitaire » (ibid. : 83), mais les processus sont similaires, que l'on s'intéresse à la réinvention des alliances à plaisanterie ou des traditions patriotiques en Inde ou en Europe. Il faut donc, là aussi, une interrogation unifiée sur le différentiel mondial de prestige des traditions nationales, et la réaction en chaîne des créations nationales par mimétisme ou ressentiment vis-à-vis de l'hégémon occidental (Kedourie 1960, 1971; Berlin 1970 ; Smith 1973; Chatterjee 1986; Greenfeld 1992 ; Crépon 1996; Amselle 2001; Jaffrelot 2005).

L'étude des conditions externes de production de ces nationalismes ne doit pas masquer la part, souvent déterminante, de l'agency interne (Galvan, Hagberg et Smith dans ce volume). Cet essor des discours de réinvention des alliances à plaisanterie constitue certes un exemple typique de la circulation nord-sud des motifs, pratiques, et narrations. L'invention des traditions locales est bien un phénomène global et cosmopolite, mais toujours co-produit par les acteurs externes et internes. Il y a là deux intentionnalités qui se rencontrent, d'une part une volonté, affirmée chez les acteurs et passeurs locaux, d'africanisation des gouvernances et, dans une optique très senghorienne ou herdérienne, de participation à l'universel par sa contribution spécifique; d'autre part, chez les acteurs internationaux, le discours bien rôdé de la "pacification» et de la 
« résolution des conflits » chez les « autres $»^{82}$. Peu importe finalement l'origine de cette "tradition" réinventée des cousinages, sauf à rester paradoxalement prisonnier du fantasme de l'« origine ». Les interprétations peuvent donc s'accorder sur l'idée que cette entreprise de promotion néo-traditionaliste n'est pas un simple calque des injonctions des organisations internationales, qui, rappelons-le, n'ont généralement aucune idée de ce dont il s'agit quand sont évoquées les « alliances à plaisanterie ", mais bien un créneau d'acteurs - les « développeurs autochtones » comme les appelle Marc Poncelet (1994) qui savent allier leurs propres stratégies de mise sur agenda et les « oripeaux du lexique international» (Mbembe 2000) dont notamment celui du « recours culturel» (Poncelet 1994).

Il n'en reste pas moins, comme le montrent Marie-Aude Fouéré et Cécile Canut, que sont en jeu dans ce discours de réinvention des alliances à plaisanterie autant les inventions nationales singulières (sénégalaise, malienne, burkinabè...) que l'image de l'Afrique en général et son rapport au reste du monde ${ }^{83}$. Ce discours fait bien partie d'un discours plus large sur la "démocratie africaine», "les droits de l'Homme» et la "diversité culturelle » (Fouéré 2005). "L'arbre à palabres » et les « cousinages à plaisanterie », redécrits et ré-interprétés à travers le vocabulaire de la démocratie et du multiculturalisme contemporain, seraient les topoi de l'Afrique et son inscription dans la mondialité, à la fois spécificités valorisées et subsumées sous le motif plus global des réquisits du Zeitgeist mondial ${ }^{84}$. Faut-il y voir un énième avatar de l'afrocentrisme renaissant (Fouéré, Canut dans ce volume) ou des logiques classiques d'appropriation et d'indigénisationendogénéisation (Hagberg, Smith, Galvan dans ce volume) ? Quoi qu'il en soit, la logique du mimétisme fonctionne à plein ${ }^{85}$.

Ce volume ne prétend pas épuiser la question, mais propose de renouveler le champ des études sur les "parentés à plaisanterie ", tombé en désuétude après son âge d'or dans l'anthropologie classique, pour interroger leurs usages politiques et contemporains qui n'avaient pas fait jusqu'à présent l'objet d'une analyse propre. Trois domaines ont été privilégiés : la dynamique des interactions en milieu urbain comme rural, le déploiement dans les espaces publics, enfin le discours de promotion de ces alliances à plaisanterie. L'investigation de ces trois dimensions a ainsi permis d'aborder les champs de recherche plus larges des relations intercommunautaires, des scènes politiques contemporaines et des processus de réinvention des traditions en Afrique de l'Ouest. La rupture avec le fonctionnalisme et l'approche ethnologique culturaliste a permis de réintroduire et d'affirmer la dimension politique présente dans les usages passés et actuels des alliances, et une interrogation des usages contemporains tant pratiques que savants.

Cependant, les limites de cette entreprise sont nombreuses. Il manque par exemple à ce volume des études sur la problématique du genre dans ces relations à plaisanterie, sur les aspects sociolinguistiques, sur les genèses historiques de ces relations... Sur ce dernier point, il reste sans aucun doute à déterminer plus précisément la matrice de ces relations dans des contextes donnés (s'il en est une qui soit commune à la variété des pratiques subsumées sous ce concept). Quelle est la part relative de l'esclavage et de la captivité, de l'islam, des institutions politiques précoloniales et notamment les pactes de sang et pratiques de serments, des stratifications sociales, du commerce de longue distance, des migrations, des conquêtes militaires, des contraintes environnementales, de l'organisation matrilinéaire, des relations avunculaires et entre cousins-croisés... ${ }^{86}$ dans la genèse des logiques pactuelles et des imaginaires afférents ${ }^{87}$ ? 
87 Par ailleurs, un des problèmes les plus délicats demeure l'entrecroisement des logiques universitaires et politico-pratiques. Pour certains chercheurs, il s'agit de dévoiler les relations de pouvoir et de domination indubitablement présentes dans ces pratiques, ou bien de souligner l'illusion irénique de l'ingénierie culturelle et les malentendus intrinsèques du néo-traditionalisme culturaliste. D’autres ont au contraire pour objectif de cartographier, de recenser, de standardiser et promouvoir ces relations, de désactiver la matrice hiérarchique en soulignant la symétrie des protagonistes ${ }^{88}$. Ces promoteurs ont donc intérêt à l'oubli des conditions de production et d'exercice de ces alliances pour promouvoir un sentiment national ou la résolution des conflits. Il apparaît que ce vaste mouvement de réinvention et de codification des traditions politiques, culturelles, religieuses dans lesquelles s'inscrit l'entreprise de promotion des cousinages, est un des aspects de la créativité politique du continent, à propos duquel le chercheur manque de recul lorsqu'il veut en évaluer l'impact. À titre provisoire, donc, l'analyse au cas par cas et les tentatives d'interprétation selon des points de vue multiples semblent être la solution la plus prudente pour analyser les ressorts, les opportunités et contraintes, les objectifs, les héritages intellectuels, la mobilisation de ressources sociales spécifiques des acteurs impliqués dans cette entreprise de promotion des cousinages. L'avenir seul dira si cette entreprise de promotion s'inscrit dans une tendance durable de réinvention politique originale du continent consécutive à la crise des grands récits (ethnologie, modernisation, développement, nationalisme, ajustement, démocratisation, bonne gouvernance) ou dans une mode passagère de célébration culturaliste d'un continent à la sociabilité supposément si « plaisante »...

Quoi qu'il en soit, la dynamique sociale des alliances à plaisanterie suscitera probablement encore longtemps les questionnements et les analyses dont les explorations dans ce volume mériteront d'être prolongées, tant leur intrication aux constructions politiques africaines les rend incontournables.

\section{BIBLIOGRAPHIE}

ABDY, D. C.

1924 « Notes on Utani and Other Bondei Customs », Man, 24 : 152-154, 164-166.

ABÉLÈS, M. \& BELLIER, I.

1996 « La Commission européenne. Du compromis culturel à la culture du compromis », Revue

française de science politique, 46 (3) : 437-456.

ABRAHAMS, R. G.

1967 The Peoples of Greater Unyamwezi, Tanzania, London, International African Institute.

AMES, D. W.

1956 « The Selection of Mates, Courtship and Marriage among the Wolof », BIFAN, Série B., 18 (1) : 156-168.

AMSELLE, J.-L.

1977 Les négociants de la savane. Histoire et organisation sociale des Kooroko (Mali), Paris, Anthropos. 
1990 Logiques métisses, Anthropologie de l'identité en Afrique et ailleurs, Paris, Payot.

1996 «L'étranger dans le monde manding et en Grèce ancienne : quelques points de comparaison », Cahiers d'Études africaines, XXXVI (4), 144 : 755-761.

2001 Branchements. Anthropologie de l'universalité des cultures, Paris, Flammarion.

2004 « Les usages politiques du passé : le N’ko et la décentralisation administrative au Mali », in

C. ERColessi \& A. TRIUlzi (eds.), State, Power and New Political Actors in Postcolonial Africa, Milano,

Feltrinelli : 7-26.

AMSELLE, J.-L. \& M’BoKolo, E. (dir.)

1985 Au cœur de l'ethnie. Tribalisme et État en Afrique, Paris, La Découverte.

ANDERSON, B.

1996 [1983] L'imaginaire national. Réflexions sur l'origine et l'essor du nationalisme, Paris, La

Découverte.

APTER, A.

1983 « In Dispraise of the Kings : Rituals “Against” Rebellion in South-East Africa », Man, 18 (3) :

521-534.

ARCIN, A.

1907 «L'organisation sociale en Guinée française. Les Familles », Revue coloniale, 46 : 18-43.

ARMSTRONG, J.

1982 Nations Before Nationalism, Chapel Hill, University of North California Press.

ARNAUD, R.

1921 « Notes sur les montagnards Habe des Préfectures de Bandiagara et de Hombori (Soudan français) », Revue d'ethnographie et des traditions populaires, $2: 241-314$.

BÂ, A. H.

1991 Amkoullel, l'enfant peul, Paris, Actes Sud.

1994 Oui mon commandant!, Paris, Actes Sud.

BÂ, 0 .

1977 Le Foûta Tôro au carrefour des cultures, Paris, L'Harmattan.

BADINI, A.

1996 « Les relations de parenté à plaisanterie : éléments des mécanismes de régulation sociale et principe de résolution des conflits sociaux au Burkina Faso », in R. OTAYEK et al. (dir.), Le Burkina Faso entre révolution et démocratie (1983-1993), Paris, Karthala : 101-116.

BALANDIER, G. \& MERCIER, P.

1952 Particularisme et évolution, les pêcheurs lébou du Sénégal, Saint-Louis, IFAN.

BARKÉ, A.

1996 « Le cousinage croisé, une institution socioculturelle en milieu africain qui participe à un mécanisme de prévention et de modération des conflits interethniques ", Annales de L'Université Abdou Moumouni de Niamey, t. V, Niamey.

BARRINGTON, L. W. (ed.)

2006 After Independence: Making and Protecting the Nation in Postcolonial \& Postcommunist States, Ann Arbor, University of Michigan Press.

BARTH, F. (ed.)

1969 Ethnic Groups and Boundaries. The Social Organisation of Culture Difference, Bergen, Universitet Forlaget; London, George Allen \& Unwin. 
BAYART, J.-F., GeschieRE, P. \& NYAMNJOH, F.

2001 « Autochtonie, démocratie et citoyenneté en Afrique », Critique internationale, 10 :177-194.

BAZIN, J.

1988 « Princes désarmés, corps dangereux. Les “rois-femmes" de la région de Ségou », Cahiers

d'Études africaines, XXVIII (3-4), 111-112: 375-442.

BEATTIE, J. H. M.

1957 « Nyoro Kinship », Africa, 27 : 317-340.

1958 « Nyoro Marriages and Affinity », Africa, $28: 1-22$.

BÉCHET, E.

1889 Cinq ans de séjour au Soudan français, Paris, Plon-Nourrit \& Cie.

BeIDELMAN, T. O.

1963 « The Blood Covenant and the Concept of Blood in Ukaguru », Africa, 33 : 321-342.

1964 « Intertribal Insult and Opprobrium in an East African Chiefdom », Anthropology Quarterly, 37

(2) : 33-52.

1966 « Utani : Some Kaguru Notions of Death, Sexuality and Affinity », Southwestern Journal of

Anthropology, 22 (4) : 354-380.

Bellagamba, A.

2002 Ethnographie, histoire et colonialisme en Gambie, Paris, L'Harmattan.

BERLIN, I.

1992 [1970] «The Bent Twig : On the Rise of Nationalism », in I. BERLIN (ed.), The Crooked Timber of Humanity: Chapters in the History of Ideas, New York, Vintage Books.

BERMAN, B., ЕYOH, D. \& KYMLICKA, W.

2004 Ethnicity and Democracy in Africa, Oxford, James Currey ; Athens, Ohio University Press.

BoILAT, D. (Abbé)

1984 [1853] Esquisses sénégalaises, Paris, Karthala.

BONTE, P.

1998 « Esclaves ou cousins : évolution du statut servile dans la société mauritanienne », in B. S

CHLEMMER (dir.), Terrains et engagements de Claude Meillassoux, Paris, Karthala : 157-182.

BOSWELL, D. M.

1969 « Personal Crisis and the Mobilization of the Social Network », in J. C. Mitchell (eds.), Social Network in Urban Situations. Analysis of Personal Relationships in Central African Towns, Manchester, Manchester University Press : 32-56.

BOULÈGUE, J.

1999 « Conflit politique et identité au Sénégal. La bataille de Bunxoy (c. 1796) », in J.-P. CHRÉTIEN \& J.-L. TRIAUd (dir.), Histoire d'Afrique. Les enjeux de mémoire, Paris, Karthala : 93-99.

BRACKELAIRE, J.-L.

1992 «Les relations de plaisanterie des Tarahumaras ", Anthropologiques, 4 : 45-60.

1993 «Changer pour rire. Les relations de plaisanterie des Tarahumaras : figure et mesure du changement ", Anthropologie et sociétés, 17 (3) : 125-140.

BRADNEY, P.

1957 « The Joking Relationship in Industry », Human Relations, 10 (2) : 179-187.

BRANT, C.

1948 « On Joking Relationships », American Anthropologist, 50 (1) : 160-162. 
BRELSFORD, V.

1935 « History and Customs of the Basala », The Journal of the Royal Anthropological Institute, 65 : 205-215.

Calame-Griaule, $\mathrm{G}$.

1954 « Les moqueries de villages au Soudan français », Notes Africaines, 61 : 12-19.

CAMARA, M.

1969 La parenté plaisante chez les Soussou de Guinée, Dakar, Enda Coorcom.

CAMARA, S.

1992 [1969] Gens de la parole. Essai sur la condition et le rôle des griots dans la société malinké, Paris, Karthala-ACCT ; Conakry, SAEC.

CANUT, $\mathrm{C}$.

2002 « Pouvoirs, places et filiation. Les Senankuya en milieu urbain au Mali », Cahiers de Praxématique, 38 : 175-197.

ChatTerjee, $\mathrm{P}$.

1986 Nationalist Thought and the Colonial World : A Derivative Discourse?, London, Zed Books.

CHRÉTIEN, J.-P.

2000 L'Afrique des Grands Lacs. Deux mille ans d'histoire, Paris, Champs-Flammarion.

ChrÉTIEN, J.-P. \& Prunier, G.

1989 Les ethnies ont une histoire, Paris, Karthala.

Christensen, J. B.

1963 « Utani : Joking, Sexual License and Social Obligations among the Luguru », American Anthropologist, 65 : 1314-1327.

Cissé, Y. T. \& КАмISSOKо, W.

1988 La grande geste du Mali. Des origines à la fondation de l'Empire, Paris, Karthala-ARSAN.

1991 Soundjata ou la gloire du Mali, Paris, Karthala-ARSAN.

Cissé, Y. T., Fofana, A. \& SAgot-Duvauroux, J.-L.

2003 La Charte du Mande et autres traditions du Mali, Paris, Albin Michel.

CNRST

1995 Plan stratégique de la recherche scientifique, Ouagadougou, Centre national de la recherche scientifique et technologique.

COLSON, E.

1953 "Clans and the Joking Relationships among the Plateau Tonga of Northern Rhodesia ", Kroeber Anthropological Society, 8-9 : 45-58 (repris in The Plateau Tonga, Manchester, Manchester University Press, 1962 : 66-83).

1968 Political Style and the Joking Relationship : The Northern Territory Legislative Council and the Darwin City Council, Presented at the Social Science Seminar, California Institute of Technology.

ConRad, D. C. \& Frank, B. E. (eds.)

1995 Status and Identity in West Africa : Nyamakalaw of Mande, Bloomington, Indiana University. CRÉPON, M.

1996 Les géographies de l'esprit. Enquête sur la caractérisation des peuples de Leibniz à Hegel, Paris, Payot \& Rivages. 
DALE, G.

1896 « Account of the Principal Customs and Habits of the Natives Inhabiting the Bondei

Country », Journal of the Royal Anthropological Institute, XXXV : 181-239.

DAVIES, C.

1990 Ethnic Humor around the World. A Comparative Analysis, Bloomington, Indiana University Press.

De BRUIJN, M. \& VAN DiJK, H.

1997 Peuls et Mandingues. Dialectique des constructions identitaires, Paris, Karthala/ASC.

DEE-GRA-REDEP

2003 Éducation à la citoyenneté, aux droits et à la paix à l'élémentaire, Dakar.

De Heusch, L.

1974 « The Debt of the Maternal Uncle : Contribution to the Study of Complex Structures of Kinship », Man, $9:$ 609-619.

DE JONG, F.

2005 « A Joking Nation : Conflict Resolution in Senegal (Casamance) », Canadian Journal of African Studies, 39 (2) : 389-413.

DelAforge, Capt.

1932 « Étude sur quelques Prénoms et Noms de Famille Bambara », Outre-Mer, IV (2-3) : 119-143.

Delafosse, M.

1912 Haut-Sénégal Niger, 3. vols., Paris, E. Larose.

DELMOND, P.

1945 « Quelques observations sur l'état-civil indigène au Soudan occidental », Bulletin de l'IFAN, 7 : 54-79.

DESAÏ, R. H.

1966 « On Joking Relationship Among the Asians of East Africa », Kampala, Makerere College, Sociology Department Working Papers, 29.

DesPlagnes, L.

1907 Le plateau central nigérien. Une mission archéologique et ethnologique au Soudan français, Paris, Larose.

DiAGNe, S. B.

1992 « L'avenir de la tradition », in M.-C. Diop (dir.), Sénégal : trajectoires d'un État, Paris, KarthalaCODESRIA : 279-300.

DiALL, G. \& TRAORÉ, B.

1999 Le cousinage à plaisanterie chez les Peul, Dakar, Enda-Coorcom.

Diallo, Y. \& SchleE, G. (dir.)

2000 L'ethnicité peule dans des contextes nouveaux. La dynamique des frontières, Paris, Karthala.

DiANÉ, A.

1997 De la modernité politique au Sénégal : rôle et place des sous-préfets dans le processus de production de la nation, Thèse de doctorat, Paris, EHESS.

Diarra, F. A. \& Fougeyrollas, P.

1969 Relations interraciales et interethniques au Sénégal, Dakar, IFAN.

DieTERLEN, G.

1951 Essai sur la religion bambara, Paris, PUF. 
1955 « Mythe et organisation sociale au Soudan français ", Journal de la Société des Africanistes, 15 (1-2) : 39-76.

1959 « Mythe et organisation sociale en Afrique occidentale ", Journal de la Société des Africanistes, 29 (1) : 119-138.

Dimitrijevic, D. (dir.)

2004 Fabrication des traditions. Invention de la modernité, Paris, Éditions de la Maison des sciences de l'Homme.

Diouf, M. M.

1996 Lances mâles. Léopold Sédar Senghor et les traditions Sérères, Niamey, CELHTO.

Diouf, B. S.

1997 L'éthique des cousinages ou l'humour au service de la paix, non publié.

Diouf, M.

1998 [1994] Sénégal, les ethnies et la nation, Dakar, NEAS.

DOKE, C. M.

1931 The Lambas of Northern Rhodesia. A Study of Their Customs and Beliefs, London, Georg G. Harrap \& Co Ltd.

DOUGLAS, M.

1968 « The Social Control of Cognition : Some Factors in Joke Perception », Man, $3: 361-376$.

DOUMBIA, P. E. N.

1936 « Étude du clan des forgerons », Bulletin du comité d'études historiques et scientifiques de l'AOF, $19: 334-380$.

Doumbia, T.

2002 « Les relations à plaisanteries dans les sociétés mandingues », Recherches africaines, janvierjuin : 28-42.

DOUYON, D.

1995 Le discours mangu chez les Dogon (Ireli). Description, analyse et fonctionnement sociologique, Thèse de doctorat, nouveau régime, Paris, INALCO.

DRIVER, H. E.

1961 Indians of North America, Chicago, University of Chicago Press.

DRUCKER-BROWN, S.

1982 « Joking at Death : The Mamprusi Grandparent-Grandchild Joking Relationship », Man, 17 (4) : 714-727.

DuBuch, $\mathrm{C}$.

1985 « Langage du pouvoir, pouvoir du langage », Politique africaine, 20 : 44-53.

DUPIRE, M.

1979 « Obscénité et société virelangues Serer (Senegal) », Research in African Literatures, 10 (1) : 75-89.

EGGAN, F.

1937 Social Anthropology of North American Indian Tribes, Chicago, University of Chicago Press.

EISENSTADT, S. N.

1956 « Ritualized Personal Relations : Blood Brotherhood, Best Friends, Compadre, etc. Some Comparative Hypotheses and Suggestions », Man, 56 : 90-95. 
EVANS-PRITCHARD, E. E.

1929 « Some Collective Expressions of Obscenity in Africa », Journal of the Royal Anthropological Institute, 49 : 311-331.

1933 « Zande Bloodbrotherhood », Africa, $6: 369-401$.

FATON, J.

1999 Du coq à l'âme, Wissous, Amok Éditions.

FATON, J. (dir.)

1998 La parenté à plaisanterie. À propos de..., Bruxelles, Atelier Graphoui.

FAY, C.

1995 «Car nous ne faisons qu'un. Identités, équivalences, homologies au Maasina (Mali) », Cahiers des Sciences humaines, ORSTOM, 31(2) : 427-456.

1997 «"Les derniers seront les premiers" : peuplements et pouvoirs mandingues et peuls au Maasina (Mali) », in M. De BRUIjN \& H. van DijK (dir.), Peuls et Mandingues. Dialectique des constructions identitaires, Paris, Karthala-ASC : 165-191.

FERME, M.

2001 The Underneath of Things, Violence, History and the Everyday in Sierra Leone, Berkeley, University of California Press.

FORREST, J. B.

2004 Subnationalism in Africa : Ethnicity, Alliances and Politics, Boulder-London, Lynne Rienner.

FORTES, M.

1945 The Dynamics of Clanship among the Tallensi, London, Oxford University Press.

1949 The Web of Kinship Among the Tallensi, Oxford, Oxford University Press.

FOUÉRÉ, M.-A.

2003 « La dimension politique de l'utani », Annuaire de l'Afrique orientale, Nairobi, IFRA.

2004 L'objet ethnologique « relations à plaisanteries » dans l'espace est-africain (Tanzanie) : de la construction savante d'une coutume à la restitution des situations sociales de l'utani, Thèse de doctorat, Paris, EHESS.

2005 « Les métamorphoses des "relations à plaisanteries". Un nouvel enjeu politique dans la construction des États-nations ", Cahiers d'Études africaines, XLV (2), $178:$ 389-430.

FREEDMAN, J.

1977 « Joking Affinity and the Exchange of Ritual Services among the Kiga of Northern Rwanda : An Essay on Joking Relationship Theory », Man, 12 (1) : 154-165.

GAILEY, H. A.

1975 Historical Dictionary of the Gambia, The Scarecrow Press Inc., Metuchen, N.J.

GAILLARD, G. (dir.)

2000 Migrations anciennes et peuplement actuel des Côtes guinéennes, Paris, L'Harmattan (« Cahiers lillois d'économie et de sociologie »).

GAMBLE, D. P.

1957 The Wolof of Senegal. Together with Notes on the Lebu and the Seerer, London, International African Institute.

GIESING, C.

2000 « Fari Sangul, Sangulé Faring. Migration et intégration politique dans le monde mandé selon les traditions des guerriers koring de la Sénégambie méridionale », in G. GAILLARD (dir.), op. cit. : 241-306. 
GIRIER, C.

1996 Parlons soninké, Paris, L'Harmattan.

GIRLING, F. K.

1957 « Joking Relationships in a Scottish Town », Man, 57 : 102.

GLUCKMAN, M.

1954 Rituals of Rebellion in South-East Africa, Manchester, Manchester University Press.

1955 Custom and Conflict in Africa, Oxford, Blackwell.

1965 Politics, Law and Ritual in Tribal Society, Oxford, Blackwell.

GOFFMAN, E.

1961 Encounters : Two Studies in the Sociology of Interaction, Indianapolis, Bobbs-Merril.

GOODALL, E. B. H.

1921 Some Wemba Words : Some Meanings and Explanations, London, Oxford University Press.

Goody, J.

1959 « The Mother's Brother and the Sister's Son in West Africa », Journal of the Royal

Anthropological Institute, $89: 61-88$.

1962 Death, Property and the Ancestors : A Study of the Mortuary Customs of the LoDagaa of West Africa, Stanford CA, Stanford University Press.

GRAVRAND, H.

1983 La civilisation sereer. Cosaan : les origines, Dakar, NEAS.

GREENFELD, L.

1992 Nationalism : Five Roads to Modernity, Harvard, Harvard University Press.

GRIAULE, M.

1948 « L'alliance cathartique », Africa, 18 (4) : 242-258.

1973 « The Mother's Brother in the Western Sudan », in P. ALEXANDRE (ed.), French Perspectives in African Studies, London, International African Institute, Oxford University Press : 11-25.

GULLIVER, P. H.

1957 « Joking Relationships in Central Africa », Man, $57: 225$.

1958 « Joking Relationships in Africa », Man, 58 : 191.

GUNDELACH, P.

2000 « Joking Relationships and National Identity in Scandinavia », Acta Sociologica, 43:113-122.

HAMMOND, P. B.

1964 « Mossi Joking », Ethnology, $3: 259-267$.

HANDELMAN, D. \& KAPFERER, B.

1972 « Forms of Joking Activity : A Comparative Approach », American Anthropologist, 74 (3) :

484-517.

HEALD, S.

1990 « Joking and Avoidance, Hostility and Incest : As Essay on Gisu Moral Categories », Man, 25 (3) : 377-392.

HERLEHY, T. J.

1984 « Ties That Bind : Palm Wine and Blood-Brotherhood at the Kenya Coast during the $19^{\text {th }}$ Century », The International Journal of African Historical Studies, 17 (2) : 285-308. 
D'HeRTEFelT, M.

1971 Les clans du Rwanda ancien. Éléments d'ethno-sociologie et d'ethno-histoire, Tervuren, Musée royal d'Afrique centrale.

HoBSBAWM, E. \& RANGER, T. (eds.)

1983 The Invention of Tradition, Cambridge, Cambridge University Press.

HoCART, A. M.

1923 « The Uterine Nephew », Man, $23: 4$.

1935 « Blood-Brotherhood », Man, 35 : 113-115.

HOFFMAN, B.

2000 Griots at War : Conflict, Conciliation and Cast in Mande, Bloomington, Indiana University Press.

HounTONDJi, P. J. (dir.)

1994 Les savoirs endogènes. Pistes pour une recherche, Dakar, Codesria ; Paris, Karthala.

VAN HOVEN, E.

1997 L'oncle maternel est roi. La formation des alliances hiérarchiques chez les Mandingues du Wuli (Sénégal), Leiden, CNWS.

HOWELL, R. W.

1973 Teasing Relationships, Addison-Wesley Module in Anthropology, 46.

HuMBLoT, P.

1918 « Du nom et des appellations chez les Malinkés des vallées du Niandian et du Milo (Guinée française) ", Bulletin du comité d'études historiques et scientifiques de l'AOF : 519-540.

IDRISSA, K.

1997 « Ethnicité, politique et démocratie au Niger », Sociétés africaines et diaspora, 5 : 45-72.

INNES, G.

1974 Sunjata : Three Mandinka Versions, London, SOAS.

1976 Kaabu and Fuladu. Historical Narratives of the Gambian Mandinka, London, SOAS.

1978 Kelefa Saane. His Career Recounted by Two Mandinka Bards, London, SOAS.

IRVINE, J.

1974 « Strategies of Status Manipulations in the Wolof Greeting ", in R. BAUMAN \& J. SHERZER

(eds.), Explorations in the Ethnography of Speaking, Cambridge, Cambridge University Press :

167-191.

1993 « Insult and Responsibility : Verbal Abuse in a Wolof Village ", in J. HILL \& J. IRVINE (eds.), Responsibility and Evidence in Oral Discourse, Cambridge, Cambridge University Press : 105-134.

JAFFRELOT, C.

2005 « For a Theory of Nationalism », in C. JAFFRELOT \& A. DieCKHOFf (eds.), Revisiting Nationalism, London, Hurst : § 1 .

JANSEN, J. \& ZOBEL, C. (eds.)

1996 The Younger Brother in Mande. Kinship and Politics in West Africa, Leiden, CNWS.

JERVIS, T. S.

1939 « A History of "Robusta" Coffee in Bukoba », Tanganyika Notes and Records, $8:$ 47-58.

JUNOD, H. A.

1912 The Life of a South-African Tribe, Neufchatel, Imprimerie Attinger Frères. 
KANE, C. H.

1961 L'aventure ambiguë, Paris, Julliard.

1995 Les gardiens du temple, Paris, Stock.

KEDOURIE, E.

1960 Nationalism, London, Hutchinson.

KedOURIE, E. (ed.)

1971 Nationalism in Asia and Africa, London, Weidenfeld \& Nicolson.

KENNEDY, J. G.

1970 « Bonds of Laughter among the Tarahumara Indians : Towards a Rethinking of Joking Relationships Theory ", in W. GoldSCHMIDT \& H. HoIJER (eds.), The Social Anthropology of Latin America, Berkeley, University of California Press.

KOMPAORÉ, P.

1999 «La parenté à plaisanterie : une catharsis sociale au profit de la paix et de la cohésion sociale au Burkina Faso ", La problématique des alliances et des parentés à plaisanterie au Burkina Faso: historique, pratique et devenir, Ouagadougou, Les Grandes conférences du ministère de la Culture, des Arts et du Tourisme.

KONATÉ, D.

1999 « Les fondements endogènes d'une culture de la paix au Mali : les mécanismes traditionnels de prévention et de résolution des conflits ", in Les fondements endogènes d'une culture de la paix en Afrique : Mécanismes traditionnels de prévention et de résolution des conflits, Paris, UNESCO.

KopytofF, I. (ed.)

1987 The African Frontier. The Reproduction of Traditional African Society, Bloomington, Indiana

University Press.

Kourouma, A.

1990 Monné, outrages et défis, Paris, Points-Seuil.

KOUYATÉ, S.

2003 Le cousinage à plaisanterie, notre héritage commun, Conakry, Éditions Ganndal.

KubA, R., LenTZ, C. \& SOMDA, C. N.

2004 Histoire du peuplement et relations interethniques au Burkina Faso, Paris, Karthala.

LABOURET, $\mathrm{H}$.

1929 « La parenté à plaisanteries en Afrique Occidentale », Africa, $2: 244-254$.

1931 Les tribus du rameau lobi, Paris, Institut d'Ethnologie.

1934 Les Manding et leur langue, Paris, Larose.

LALLEMAND, S.

1975 «"Têtes en loques" : insulte et pédagogie chez les Mossi », Cahiers d'Études africaines, XV (4), 60 : 649-667.

1977 «Relations à plaisanterie, relations froides », in Une famille mossi, Paris, Ouagadougou, CNRSCVRS, Recherches Voltaïques, 17 : 269-294.

LANDSBERG, P. W.

1977 Kinship and Community in a Tanzanian Coastal Village, Ph. D. Dissertation, Davis, University of California.

LAUNAY, R.

1977 « Joking Slavery », Africa, 47 (4) : 413-422. 
1995 « The Dieli of Korhogo. Identity and Identification », in D. C. CONRAD \& B. E. FrAnK (eds.), Status and Identity in West Africa. Nyamakalaw of Mande, Bloomington-Indianapolis, Indiana University Press : 153-169.

LEENHARDT, M.

1930 Notes d'Ethnologie Néo-calédoniennes, Paris, Institut d'ethnologie.

1947 Do kamo : la personne et le mythe dans le monde mélanésien, Paris, Gallimard.

LEGRÉ OKOU, H.

1994 Les conventions indigènes et la législation coloniale, Abidjan, Éditions Neter.

LERICHE, A.

1956 « Anthroponymie toucouleur », BIFAN, 18 (1-2) : 169-185.

Leroy, A. \& BALDE, A. O. K.

2003 Parlons poular. Peul du Fouta Djalon, Paris, L'Harmattan.

LESLIE, J. A. K.

1963 A Survey of Dar-Es-Salaam, Oxford, Oxford University Press.

LEWIS, R.

1954 Sierra Leone : A Modern Portrait, London, Her Majesty's Stationnary Office.

LEYNAUD, E. \& CISSÉ, Y. T.

1978 Paysans malinke du Haut-Niger, Bamako, Imprimerie Populaire du Mali.

LONG, S.

2005 « À quoi joute-t-on ? Devinettes tonales chez les Moose », ethnographiques.org, 7.

LOWIE, R. H.

1912 « Social Life of the Crow Indians », Anthropological Papers of the American Museum of Natural History, 9 (2) : 179-253.

1917 " Notes on the Social Organization and Customs of the Mandan, Hidatsa and Crow Indians ", Anthropological Papers of the American Museum of National History, 21 (1) : 97-99.

LUCAS, S. (ed.)

1974 Utani Relationships in Tanganyika, Université de Dar-es-Salaam, 7 vol.

LUNDBERG, C. C.

1969 « Person-focused Joking : Pattern and Function », Human Organization, 28 : 22-28.

LUOMALA, K.

1966 « Numbskull Clans and Tales : Their Structure and Function in Assymetrical Joking Relationships ", in J. GREENWAY (ed.), The Anthropologist Looks at Myth, Austin, University of Texas Press : 157-198.

LY, B.

1966 L'honneur et les valeurs morales dans les sociétés ouolof et toucouleur du Sénégal : étude de sociologie, Thèse de doctorat, 2 vol., Paris, FLSH.

Mac AlPine, A. G.

1906 « Tonga Religious Beliefs and Customs », Journal of the Royal African Society, 18 : 264-267.

MARIKO, K. A.

1990 « La parenté à plaisanteries comme facteur d'intégration sociale en Afrique occidentale ", Colloque international, Aires culturelles et création littéraire en Afrique, Biennale des arts et des lettres, Dakar, 12-18 décembre, NEAS-ACTT : 35-38. 
MAUSS, M.

1928 « Parentés à plaisanterie », Paris, Annuaire de l'École pratique des hautes études, Section des sciences religieuses (« Les classiques en sciences sociales »), Melun, Imprimerie administrative : 3-21.

MAYER, P.

1950 « The Privileged Obstruction of Marriage Rites among the Gusii », Africa, 20 :113-125.

1951 « The Joking of "Pals" in Gusii age-sets », African Studies, $10: 27-41$.

MBEMBE, A.

1996 « La "chose" et ses doubles dans la caricature camerounaise ", Cahiers d'Études africaines, XXXVI (1-2), 141-142:143-170.

2000 De la postcolonie. Essai sur l'imagination politique dans l'Afrique contemporaine, Paris, Karthala.

MCKAY, W. F.

1975 A Precolonial History of the Southern Kenya Coast, Ph. D. Thesis, Boston University.

McNAughton, P. R.

1988 The Mande Blacksmiths: Knowledge, Power and Art in West Africa, Bloomington, Indiana University Press.

McVICAR, Rev.

1935 « Sibs, Privileged Familiarity and Cross-Cousin Marriage among the Waluguru », Primitive Man, 8 : 57-67.

MEAD, M.

1934 « Kinship in the Admiralty Islands », Anthropological Papers of the American Museum of Natural History, 34 (2) : 189-358.

MEEK, C. K.

1925 The Northern Tribes of Nigeria : An Ethnographical Account of the Northern Provinces of Nigeria Together with a Report on the 1921 Decennial Census, 2 vol., London, Oxford University Press.

MeIllassoux, C.

1978 « Rôle de l'esclavage dans l'histoire de l'Afrique occidentale », Anthropologie et Sociétés, 2 (1) : 117-148.

MEÏTÉ, M.

2004 « Les alliances à plaisanteries comme voie », Éthiopiques, 72.

MELLAND, F. H.

1967 [1923] In Witch-Bound Africa. An Account of Primitive Kaonde Tribe and Their Beliefs, London, Franck Cass \& Co. Ltd.

MitCHELL, C.

1956a The Yao Village: A Study in the Social Structure of a Malawian Tribe, Manchester, Manchester University Press.

1956b « The Kalela Dance, Aspects of Social Relationships among Urban Africans in Northern Rhodesia ", The Rhodes-Livingstone Papers, 27, The Rhodes-Livingstone Institute, Manchester University Press : 35-44.

MKANGI, K.

1997 Indigenous Social Mechanisms of Conflict Resolution in Kenya : A Contextualized Paradigm for Examining Conflict in Africa, Nairobi, University of Nairobi. 
MoLINIÉ, Lt

1959 Les systèmes patronymiques au Soudan Central, Centre militaire d'information et de spécialisation pour l'Outre-Mer, $4^{\mathrm{e}}$ trimestre, 544/A.

MONENEMBO, T.

2004 Peuls, Paris, Éditions du Seuil.

MONTEIL, L.-P.

1894 De Saint-Louis à Tripoli par le Lac Tchad. Voyage au travers du Soudan et du Sahara accompli pendant les années 1890-91-92, Paris, Félix Alcan.

MONTEIL, C.

1903 Monographies de Djenné, cercle et ville, Tulle, Imprimerie Jean Mazeyrie.

1915 Les Khassonké. Monographie d'une peuplade du Soudan français, Paris, Ernest Leroux.

1924 Les Bambara du Ségou et du Kaarta : Étude historique, ethnographique et littéraire d'une peuplade du Soudan français, Paris, Larose.

MOREAU, R. E.

1941 « The Joking Relationship (utani) in Tanganyika », Tanganyika Notes and Records, 12 :1-10.

1944 « Joking Relationships in Tanganyika », Africa, 14 (7) : 386-400.

MUDIMBE, V. Y.

1988 The invention of Africa. Gnosis, Philosophy and the Order of knowledge, Bloomington, Indiana University Press.

MuRPhy, W. P. \& Bledshoe, C. H.

1987 « Kinship and Territory in the History of a Kpelle Chiefdom (Liberia) », in I. KopyToff (ed.), The African Frontier. The Reproduction of Traditional African Societies, Bloomington, Indiana University Press : 121-147.

MWOROHA, E.

1977 Peuples et rois de l'Afrique des lacs, Dakar, NEA.

MYAMBA, F.

2000 Joking Relationship as Peace Enhancement in the Society. Case Study : Njombe, Iringa, Tanzania, Dissertation, The University of Dar-es-Salaam.

2001 Peace and Culture : A Case of Reproduction and Articulation of Utani Among the Bena Ethnic Group in Tanzania, Dar-es-Salaam, Ministry of Education and Culture.

N'DiAYE, B.

1970a Les castes au Mali, Bamako, Éditions populaires.

1970b Groupes ethniques au Mali, Bamako, Éditions populaires.

NDIAYE, R.

1992 «Correspondances ethno-patronymiques et parenté plaisantante : une problématique d'intégration à large échelle », Environnement africain, 8 (3-4) : 97-128.

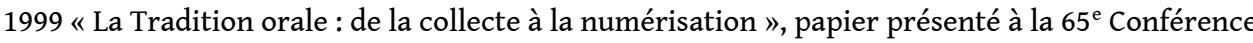
générale de l'International Federation of Library Associations, 20-28 août, Bangkok.

2000 « La Sinankuya : la parenté plaisante comme moyen de résolution des conflits », Colloque sous-régional, Parenté plaisante, citoyenneté et culture de la paix, 24-27 janvier, Dakar, Cercle de l'Union.

2001 « Parenté à plaisanterie, citoyenneté et culture de la paix en Afrique de l'Ouest », Colloque sous-régional des Églises, Réconciliation et charité, 28 mars, Dakar. 
NDIAYE, S. W.

1999 Un pont de lumière pour le fleuve, Dakar, NEAS.

NEWBURY, D.

1991 Kings and Clans : Ijwi Island and the Lake Kivu Rift, 1780-1840, Madison, The University of Wisconsin Press.

NiANE, D. T.

1960 Soundiata ou l'épopée mandingue, Paris, Présence africaine.

Nicolaisen, J.

1963 Ecology and Culture of the Pastoral Tuareg. With Particular Reference to the Tuareg of Ahaggar and Ayr, Copenhague, National Museum.

NYAMBA, A.

1999 « La problématique des alliances et des parentés à plaisanterie au Burkina Faso : historique, pratique et devenir ", Les Grandes conférences du ministère de la Communication et de la Culture du Burkina Faso, Ouagadougou : 73-91.

2001 « Les relations de plaisanteries au Burkina Faso : un mode de communication pour la paix sociale ", Communication, 21 (1) : 119-140.

O'BANNON, B. R.

2003 Do Not Speak to Me of Cousinage: Neoliberalism, Changing Identities and Conflict Management in Senegal, Paper presented at the African Studies Association, october, Annual Meeting, Boston.

PAGEARD, R.

1958 « Notes sur les rapports de "senankouya" au Soudan français, particulièrement dans les cercles de Ségou et de Macina ", Bulletin de l'IFAN, XX, série B : 123-141.

1959a « Note sur les Diawambé ou Diokoramé », Journal de la Société des Africanistes, 29 (2):

239-260.

1959 b « Note sur les Kagoro et la chefferie de Soro », Journal de la Société des Africanistes, 29 (2) :

261-271.

Palau-Marti, M.

1960 « Conduites abusives permises en Afrique », Bulletin de l'IFAN, XXII : 299-327.

PÂQUES, V.

1954 Les Bambaras, Paris, PUF.

PARKIN, D.

1980 « The Creativity of Abuse », Man, $15: 45-64$.

Paulme, D.

1939 « Parenté à plaisanteries et alliance par le sang en Afrique occidentale », Africa, XII (4) :

433-444.

1968 « Pactes de sang, classes d'âge et castes en Afrique noire ", Archives européennes de Sociologie, $9(1): 12-33$.

PEDLER, M. F. J.

1940 « Joking Relationship in East Africa », Africa, 13 : 170-173.

Peek, P. M. \& YANKah, K.

2003 African Folklore. An Encyclopedia, New York, Routledge.

PÉLISSIER, P.

1966 Les paysans du Sénégal. Les civilisations agraires du Cayor à la Casamance, Saint-Yrieix, Imprimerie Fabrègue. 
PERINBAM, B. M.

1997 Family Identity and the State in the Bamako Kafu, c.1800-c.1900, Boulder, Westview Press.

PERSON, Y.

1968 Samory. Une révolution Dyula, 2 vol., Nîmes, Imprimerie Barnier.

POLLET, E. \& WinTER, G.

1972 La société Soninké (Dyahunu, Mali), Bruxelles, Édition de l’Université de Bruxelles.

PONCELET, M.

1994 Une utopie post-tiermondiste. La dimension culturelle du développement, Paris, L'Harmattan.

POUWER, J.

1964 « Radcliffe-Brown's Ideas on Joking Relationships Tested by Data from Mimika », International Archives of Ethnography, $1: 11-30$.

PRIGNITZ, G.

2001 « La mise en scène du plurilinguisme dans l'œuvre de Jean-Hubert Bazié. Une représentation de la situation sociolinguistique du Burkina », Cahiers d'Études africaines, XLI (3-4) : 795-814.

RADCLIFFE-BROWN, A. R.

1924 « The Mother's Brother in South Africa », South African Journal of Science, 21 : 542-555.

1940 « On Joking Relationships », Africa, 13 (3) : 195-210.

1949 « A Further Note on Joking Relationships », Africa, $19: 133-140$.

1968 [1952] Structure et fonction dans la société primitive, Paris, Éditions de Minuit.

RADIN, $\mathrm{P}$

1923 The Winnebago Tribe, Lincoln, University of Nebraska Press.

RENAN, E.

1947 [1882] « Qu'est-ce qu'une nation? », Conférence à la Sorbonne du 11 mars 1882, in H. P SICHARI (dir.), CEuvres complètes d'Ernest Renan, t. I, Paris, Calmann-Lévy : 887-906.

REYNOLDS, V.

1958 « Joking Relationships in Africa », Man, $58: 21$

RICHARDS, A. I.

1935 « Preliminary Notes on the Babemba of North-Eastern Rhodesia », Bantu Studies, 9 (3) :

225-253.

1937 « Reciprocal Clan Relationships among the Bemba of N.E. Rhodesia », Man, 37 : 188-193.

RIESMAN, P.

1974 Société et liberté chez les Peuls Djelgôbé de Haute-Volta. Essais d'anthropologie introspective, Paris, Mouton.

RIGBY, P.

1968 « Joking Relationships, Kin Categories and Clanship among the Gogo », Africa, 38 : 133-155.

RODEGEM, F. M.

1976 « Les injures agonistiques des gardiens de bétail au Burundi », Psychopathologie africaine, XII (3) : 413-442.

SALAMONE, F. A.

1979a « Children's Games as Mechanisms for Easing Ethnic Interaction », Anthropos, 74 : 201-210. $1979 b$ « Dukawa-Kamberi Relationships of Privileged Familiarity : Implications for the Theory of Play », Ethnicity, 6 (12) : 123-136. 
SAMBOU, $\mathrm{S}$.

2005 Aguène et Diambone, Dakar, Niamagne Édition.

SARR, S.

2000 « L'Afrique, un défi pour l'éducation à la paix », Conférence à Lomé.

SCHAFFER, M. \& COOPER, C.

1980 Mandinko. The Ethnography of a West African Holy Land, New York, Holt, Rinehart and Winston.

SCHAFFER, M.

2003 Djinns, Stars and Warriors. Mandinka Legends from Pakao, Senegal, Leiden, Brill.

SCHIAVONE, C.

2001 La parole plaisante nel romanzo senegalese postcoloniale, Roma, Bulzoni.

SCHILDKROUT, E.

1975 « Ethnicity, Kinship and Joking among Urban Immigrants in Ghana », in B. DU ToIT \& H. SAFA (eds.), Migration and Urbanization: Models and Adaptive Strategies, The Hague, Mouton : 245-263. 1978 People of the Zongo: The Transformation of Ethnic Identities in Ghana, Cambridge, Cambridge University Press.

SCHOTTMAN, W.

1998 « The Joking Relationship as Practised by the Batoombu of Benin », in E. BOESEN, C. HARDUNG, R. KUBA (dir.), Regards sur le Borgou : pouvoir et altérité dans une région ouest-africaine, Paris,

L'Harmattan : 155-174.

SCRIVENOR, T. V.

1937 « Some Notes on Utani, or the Vituperative Alliances Existing between the Clans in the Masasi District », Tanganyika Notes and Records, 4 : 72-74.

SESSOUMA, D.

1993 « Les parentés à plaisanterie », Regard, $37:$ 8-11.

SHARMAN, A.

1969 " "Joking" in Padhola : Categorical Relationships, Choice and Social Control », Man, 4 (1) : 103-117.

SIRAN, J.-L.

2006 « Idéal du mariage et plaisanterie ", L’Homme, à paraître.

SISSAO, A. J.

2002 Alliances et parentés à plaisanterie au Burkina Faso : mécanismes de fonctionnement et avenir, Ouagadougou, Sankofa \& Gurli Éditions.

SISSOKO, F. D.

1950 « L'humour africain », Présence Africaine, 8-9 : 227-239.

SMITH, P.

1973 « Principes de la personne et catégories sociales », in La notion de personne en Afrique Noire, Paris, Éditions du CNRS.

SMITH, A. D.

1983 Theories of Nationalism, London, Duckworth.

SMITH, É.

2004 « Les cousinages de plaisanterie en Afrique de l'Ouest, entre particularismes et universalismes », Raisons politiques, 13 : 157-169. 
Smith, E. W. \& DALE, A.

1920 The Ila-speaking Peoples of Northern Rhodesia, London, McMillan.

DE SOUSBERGHE, L.

1955a Structures de parenté et d'alliance d'après les formules Pende (ba-Pende, Congo belge), Bruxelles, Académie

\section{NOTES}

1. «Democratic Mali Sets an Example », State Magazine, $n^{\circ} 467$, pp. 10-15, April 2003. State Department, United States of America.

2. T. S. DiALlo, « À propos du pacte national », Guinea Forum, <http://www.guineaforum.org/Analyses/index.asp ?ana=357\&Lang=F>.

3. Macky SALL, Premier ministre du Sénégal, Discours de politique générale, 20 octobre 2004.

4. B. A. BEOGO, « Burkina Faso, terre bénie », in L'Opinion, n 390, dossier Journée nationale de Pardon, 23-29 mars 2005.

5. Abdrahamane SALIOU, « Élections présidentielles : les raisons du succès ! ", Haske, 21 novembre 2004.

6. <http://www.everyculture.com/To-Z/Zambia.html>.

7. Colloque tenu au Centre d'études et de recherches internationales (CERI) à Paris les 27 et 28 octobre 2005, dont ce numéro reproduit certaines contributions. Cette thématique a également été abordée lors de trois manifestations récentes : un atelier tenu au Max Planck Institute for Social Anthropology (Halle), intitulé Friendship, Descent, Alliance in Africa, le 18 décembre 2002 ; lors d'un atelier au meeting annuel de l'African Studies Associations de 2003 à Boston, intitulé Joking Kinship and Interethnic Cooperation in Senegambia, et un séminaire de l'Institut d'études africaines d'Aix-en-Provence, intitulé Pardon, palabre, plaisanterie : espaces publics africains et passés recomposés, le 16 novembre 2005.

8. Le terme est entre guillemets car le processus de valorisation et de re-découverte des « traditions » d'alliances à plaisanterie est indissociablement local et global, national et cosmopolite, africain et mondial. Les inventions de la tradition (HOBSBAWM \& RANGER 1983) sont généralement le co-produit de logiques internes et externes, que ce soit la production des nationalismes européens ou celle des divers afrocentrismes. On se gardera donc ici de toute caricature de ces processus en termes d'invention exogène ex nihilo ou de son pendant indigéniste d'agency purement locale.

9. Cette distinction faite par RADCLIFFE-BROWN (1940) entre relation symétrique (égalitaire) et asymétrique (inégalitaire) est critiquable, car il existe en fait tout un continuum possible entre la symétrie et l'asymétrie.

10. C'est pourquoi l'on préfère utiliser le terme d' « alliance à plaisanterie » que celui de « parenté à plaisanterie », selon la distinction faite par Sory CAMARA (1992 : 39).

11. On peut ainsi relever, par exemple, comme termes utilisés pour décrire les alliances à plaisanterie ou des " pactes » plus formalisés, les termes senankuya et jo en bamanakan, njongu et kallengoraaxu en soninke, kal et gàmmu en wolof, dendiraagu njongu (ou dendiragaal) et hoolaare en pulaar, sanawuya et dankutoo en maninkakan, sanakuyagaal en pulaar du Fuuta-Jalon, maasir en serer, agilor en joola, rakiire en moore, moal en lobi, díkale en bissa, mangu en dogon, bariwa en bwaba, baaseterey en songoy, ubuse en kinyarwanda, 
utani en kiswahili, bukulo en gisu, banungwe en bemba, awalongo en lamba, uzukuru en nyanja, mwilo en yao, wusensi en lunda, muzenze en kaonde, etc. Il existe également des termes dérivés, désignant les partenaires de la relation, le verbe d'action, etc.

12. Par exemple, « suzeraineté » (BÉCHET $1889: 186$ ), « parentés réciproques » (HUMBLOT 1918 : 520), « parenté à libre parler » (LEENHARDT 1930 : 86 ; BA 1977 : 196), « alliance cathartique » (GRIAULE 1948), « privautés domestiques » (PÉLISSIER $1966: 194)$, « injure affectueuse » (DiARRA \& FougeYRollas $1969: 8$ ), « cousinage rituel » (GRAVRAND $1983: 154$ ), « parenté plaisantante » (NDIAYE 1992), « pacte de paix perpétuelle »(Diouf $1996: 15)$, « cousinage à plaisanteries » (KOUYATÉ 2003). En anglais : " privileged familiarity » (LOWIE 1917 : 42 ; MC VICAR 1935), « comradeship-in-sport » (WILSON-HAFFENDEN 1930 : 117s), « playmateship » (MEEK 1925 : 31-32), « vituperative alliances » (SCRIVENOR 1937), « funeral friendship » (STEFANISZYN 1950), « clan jest » (STEFANISZYN 1951), « intertribal insult » (BEIDELMAN 1966), « teasing relationships » (HowELL 1973), « joking affinity » (F REEDMAN 1977), « teasing kinship » (UNESCO $1974:$ 19), « joking partnership » ou « affinal joking » (SCHILDKROUT $1978: 153)$...

13. Dans le discours émique et médiatique, en français, les termes foisonnent également : « injures diplomatiques ", « fraternités à plaisanterie », « fraternités interethniques », « ethnies à plaisanterie », « railleries ethniques », « cousinages ethniques » " plaisanteries de cousinage ", " plaisanteries de parenté ", " pseudo-parenté ", " parenté par plaisanterie », « cousinages béats »... Les usages diffèrent selon les pays et les locuteurs : " parenté à plaisanterie » est davantage utilisé au Burkina Faso par exemple, alors qu'au Sénégal c'est plutôt « cousinage à plaisanterie » ou " cousinage ».

14. «Joking kinship », plus proche du terme français, est le terme désormais utilisé, de préférence à "joking relationship " (voir panel ASA, Boston, 2003 et les contributions de Dennis Galvan et Mark Davidheiser dans ce volume).

15. Marie-Aude FouÉRÉ (2004 : 34-36) souligne la mention faite de simulacres de combats pour la préséance entre caravanes de l'Est africain par Burton et Speke, passablement étonnés, dans leurs journaux de voyage respectifs en 1858. Pour ABRAHAMs (1967 : 66) qu'elle cite, il s'agirait d'une sorte de proto-utani.

16. L'analyse de LABOURET est la plus complète après Delafosse - qu'il cite - embrassant toute la sous-région, des Joola de Casamance au Gurunsi de Haute-Volta. Il prolongera cette analyse dans Les tribus du rameau lobi $(1931$ : 248) et Les Manding et leur langue (1934: 98-104).

17. On n'évoque pas ici les nombreux travaux comparatifs de sociologie et d'anthropologie sur la « fonction » du rire et de l'humour qui ont longuement discuté la théorie de Radcliffe-Brown. Ceux de LUNDBERG (1969) et Howell (1973) sont fréquemment cités dans les travaux africanistes.

18. Pour une lecture critique des différentes monographies en Tanzanie, ainsi que des écrits antérieurs de MCVICAR (1935), SCRIVENOR (1937), PEDLER (1940) et MOREAU (1941, 1944), voir FouÉRÉ (2004).

19. En 1974, lors du meeting de l'African Studies Association à Chicago, s'est tenu un atelier sur les joking relationship, incluant six contributions dont certaines sont devenues des articles : SCHILDKROUT 1975 ; FREEDMAN 1977 ; STEVENS 1978 ; SALAMONE $1979 \mathrm{~b}$.

20. Il n'est donc pas étonnant de trouver dans un dictionnaire du « folklore africain », un article sur les joking relationship (РеEK \& YANKAH 2003).

21. On s'intéresse ici principalement à l'Afrique de l'Ouest francophone, mais les études sur des terrains ghanéens ou nigérians n'ont pas manqué (GOODY 1959, 1962 ; STEVENS Jr 
1973, 1978 ; SCHILDKROUT 1975 ; SALAMONE 1979a, 1979b ; DRUCKER-BROWN 1982 ; WeGRU

2000).

22. La recension de Molinié (1959) est la plus aboutie. Elle se présente comme un annuaire des jamuw, véritable répertoire alphabétique classant pour chaque jamu ses senankuw, ses équivalents dans les autres communautés, son terroir d'origine, son tana (interdits), le mythe d'origine, etc.

23. Marie-Aude FoUÉRÉ (2004 : 75-79) a bien souligné la prégnance du modèle jural dans la théorisation des relations à plaisanterie par les anthropologues.

24. On note cependant que le CODESRIA et l'ouvrage programmatique sur les « savoirs endogènes ", édité par Paulin HounTONDJI (1994) ne se sont pas emparés de cette thématique.

25. Un exemple parmi d'autres, pris dans le champ journalistique ivoirien : « La parenté à plaisanteries : quel impact dans une Côte-d'Ivoire en crise ? ", Le Patriote, 13 octobre 2003. 26. Cette question complexe de la hiérarchie et de la domination est traitée plus loin dans l'introduction.

27. Le Quotidien, 27 mai 2004.

28. Au-delà de ces éléments de contexte global, chacune des scènes nationales étudiées dans ce volume a ses dynamiques internes, évoquées par les auteurs, qui ne sont pas sans conséquences sur la saillance de la problématique des alliances à plaisanterie : le conflit casamançais au Sénégal, la crise politique de l'après Zongo au Burkina Faso, la décentralisation et le développement du tourisme au Mali, etc.

29. On peut faire des remarques similaires concernant l'espace est-africain. CHRISTENSEN (1963 : 1323), discutant les études de MorEAU (1941) et SPIES (1943), souligne que les relations d'utani ont pour origines la guerre, le commerce longue-distance, l'esclavage et, parfois, « l'acte du bon samaritain » (SPIES $1943: 49$ ).

30. Pour Tal TAMARI (1997 : 133), le lien entre senankuya et « castes » est explicite, car elle fait l'hypothèse que « la relation entre nobles et gens de caste aurait [...] pu naître d'une alliance à plaisanterie [...] scellée dans des conditions d'inégalité particulièrement marquées ». Sur ce point, voir PAULme 1939, 1968 ; CAMARA 1969, 1992 : 38-50 ; AMSELLE 1977 : 37-48 ; LAUNAY 1995 : 161 ; TAMARI 1997 : 131-138. Dans le cas de la senankuya entre Peuls et forgerons (DouMBIA 1936), ou entre certains patronymes et certains groupes professionnels, l'analogie est encore plus flagrante.

31. Ainsi, des plaisanteries entre Toucouleur et Serer sur les causes du « départ » des Serer du Tekrur rapportées par les traditions orales (refus de l'islamisation ou fuite d'esclaves), entre Joola et Serer à propos du mythe d'Ageen et Jamboñ (qui était « devant » et qui était « derrière » dans la pirogue de la légende des deux sœurs), entre Peuls et forgerons (qui a fait advenir l'autre à la condition d'homme), entre Peuls et Jaaxanke (qui a capturé l'autre), entre Peuls et Malinké sur la chute de Kansala (quelle version de la bataille imposer), etc. Derrière ces mémoires apparemment divergentes, c'est tout de même une même matrice politico-culturelle faite d'oralité et de récits à large diffusion dans toute la sous-région qui se retrouve. Pour un exemple de la construction de mémoires partagées de la bataille de Bunxoy entre le Kajoor et le Fuuta, voir BoulÈGUE 1999.

32. En effet, l'esclavage domestique précolonial semble constituer une des matrices des relations de cousinage à plaisanterie observées actuellement (LAUNAY 1977). Le langage atteste de l'héritage de la servilité dans les imaginaires et de la transition délicate du statut d'« esclave » (bien réel) à celui de « cousin ». Ainsi, en 1954, un chef vaï de Sierra- 
Leone peut répondre à son interlocuteur qui lui demande si l'esclavage est encore pratiqué : « No, we don't have slaves now - we have cousins » (rapporté sans précision sur le contexte de l'énonciation par LEWIs (1954 : 67, italiques de Lewis). Sur les résonances de l'esclavage dans les métaphores de la parenté en Sierra Leone, voir M. FERME (2001 : 82-88). Sur le difficile et incertain passage des harâtin du statut d'« esclave » à celui de « cousin » (notamment par le mariage) de leurs anciens maitres dans la société maure, voir BONTE (1998 : 170-182). On note d'ailleurs que les premières mentions des alliances à plaisanterie dans la littérature des voyageurs ou des administrateurs coloniaux laissent planer une ambiguïté quant au caractère métaphorique ou réel de l'esclavage entre «alliés ». TAUTAIN (1885: 8) comme LABOURET (1929: 251) insistent ainsi sur l'asymétrie radicale et l'unilatéralité de ces « alliances » pensées par analogie avec l'esclavage. On trouve par ailleurs, dans le récit de voyage de Parfait-Louis MonTEIL (1894: 99s), un exemple saisissant d'une « capture » rhétorique de villageois bobo par un griot peul, qui en fait ses « esclaves » et les oblige à un acte de soumission envers Monteil. Il ne semble pas s'agir à l'époque d'une quelconque alliance à plaisanterie entre Peuls et Bobo comme elle se pratique actuellement...

33. Point déjà souligné par David PARKIN (1980).

34. Cela n'implique pas pour autant d'adhérer à l'hypothèse cathartique ou à une analyse du type de celle de Max GluckMAN (1954) en termes de « rituels de rébellion ». Pour une analyse du rituel Ncwala (présenté par Gluckman comme le rituel de rébellion par excellence), comme une forme de relation à plaisanterie, voir APTER (1983) qui tente de réconcilier les approches de Gluckman, Beidelman et Radcliffe-Brown.

35. « Undoubtedly easier communications are breaking down the observances. As between the Chagga and Pare the forfeit customs are said to be a thing of the past because intercourse is so frequent. Among the Bondei the "joking" is obsolescent. It seems impossible that Zigua living on the main Turiani-Korogwe road could keep open house for the Nyamwezi, the Sukuma, the Kami, the Luguru, the Hehe, the Gogo, and the Zaramu who now pass in such numbers on their way to work on the sisal estates. And it is obvious that a place like Tanga market, with its admixture of tribes, would be in constant uproar if mutual recrimination, "April fooling", and forfeiting went on with pristine vigour. Other Western influences must have a damping effect... » (MOREAU 1944 : 398-399). 36. À ce compte-là, qu'est-ce qui différencie ces pratiques de plaisanterie de celles, par exemple, entre commissaires européens que rapportent Marc ABÉLÈs et Irène BELLIER (1996)?

37. Les labels ethniques et patronymiques étant labiles et manipulables, il est très fréquent qu'un individu se fasse passer pour un " cousin à plaisanterie » afin de bénéficier des avantages attendus de la relation (demander un service, bénéficier de dons lors de cérémonies, profiter de l'hospitalité, échapper à une sanction, faire basculer une interaction ou une négociation à son avantage, sauver sa vie, tenter de gagner des électeurs, etc.). Au-delà de ces instrumentalisations jugées illégitimes et abusives une fois découvertes, les alliances à plaisanterie sont fréquemment utilisées dans le cadre de pratiques professionnelles pour faciliter le contact, faire passer un message ou obtenir des renseignements (agent de recensement, personnel médical, commerçant, facteur, enseignant, chercheur, agent d'encadrement agricole, personnel d'ONG ou personnel administratif en tournée de sensibilisation, politicien, magistrat, policier, etc.).

38. Chanson Sordassi kè, Album Kéléa, s.d. 
39. Dans la chanson Bara bali, à travers un dialogue avec son guitariste Petit Condé, l'artiste dénonce la fainéantise de la plupart des patronymes (KouYATÉ 2003 : 39). Condé et Diabaté, comme leurs équivalents Koné et Traoré, sont senankuw.

40. Sous la forme d'une émission culturelle invitant un érudit chargé de parler de ces alliances et d'en faire la liste, ou bien tout simplement sous forme ludique, notamment par le biais des appels des auditeurs.

41. Chez certains auteurs comme Amadou Hampathé $\mathrm{Ba}$, la logique de patrimonialisation est très présente, car ces usages sont toujours suivis de notes de bas de pages ou de parenthèses pour expliquer ces pratiques au lecteur non initié. Chez d'autres auteurs (Tierno Monenembo, Ahmadou Kourouma, Jean-Hubert Bazié), l'usage des alliances à plaisanterie dans le roman est d'ordre plutôt ludique, faisant partie du décor culturel n'ayant pas besoin d'être expliqué.

42. À l'inverse, tout au long du roman, les Peuls sont moqués selon le point de vue des Serer.

43. Les forums sénégalais, maliens, et guinéens ont notamment été consultés. On note qu'avec le support écrit, l'ambiguïté du « cadre » de l'interaction est accrue et donne souvent lieu à des malentendus et polémiques. Voir également le débat sur un forum camerounais sur la possibilité ou non de l'importation de la pratique « burkinabè » de la parenté à plaisanterie au Cameroun : « Les moqueries inter-tribus au Cameroun... est-ce possible ? » (sic).

44. Qui ne se limite évidemment pas à la pratique des alliances à plaisanterie. La dérision populaire du pouvoir autoritaire a largement été analysée dans le cadre des analyses du « politique par le bas » (TOULABOR 1981 ; DUBUCH 1985 ; MBEMBE 1996, 2000 : 139-186) mais jusqu'à présent le comique populaire puisant dans le registre des alliances à plaisanterie (HAGBERG et DOUYON dans ce volume) n'avait pas été étudié.

45. Ainsi dans les années 1980, certains auditeurs de l'émission Rions un peu de l'ORTM au Mali ont pu se plaindre que les blagues visaient trop exclusivement une certaine communauté. De même, sur des forums Internet sénégalais ou guinéens, des remarques sont faites en ce sens par les internautes. C'est toute l'ambiguïté du « cadre » de l'interaction, les blagues dépréciatives n'étant pas limitées aux échanges plus ou moins codifiés et réciproques du cousinage. Il suffit à cet égard de rappeler la prégnance funeste de certains stéréotypes au Rwanda ou en Côte-d'Ivoire par exemple, qui ont aussi emprunté le canal de « blagues » radiodiffusées qui faisaient rire y compris les groupes visés...

46. Le Quotidien, 24 juillet 2005.

47. Ainsi, les jugements posthumes portés sur Senghor au Sénégal ou Lamizana au Burkina Faso.

48. Au Burkina Faso, Alain SISSAO (2002 : 141) appelait ainsi de ses vœux un « commandement apaisé », qui « permettait de mieux comprendre les populations administrées » à l'image de la nomination d'administrateurs cerma en pays lobi et inversement. Au Sénégal, la question s'est posée notamment à propos du conflit en Casamance dont certaines lectures en termes d' « erreurs » ou d'« abus » du commandement territorial ont un temps justifié la nomination d'administrateurs serer en pays joola. Certains auteurs ont affirmé que gouverneurs et préfets au Sénégal étaient parfois délibérément nommés dans des régions où ils comptent de nombreux cousins (V ILLALON 1995 : 54 ; DiANÉ 1997 : 360 ; DE JONG 2005 : 398). Cela semble s'être effectivement pratiqué, mais ne constitue absolument pas une règle générale. Quoi qu'il en soit, cette utilisation (réelle ou réclamée) des liens d'alliance à plaisanterie entre communautés par 
l'administration républicaine au nom d'une efficacité supposément accrue soulève des questions intéressantes quant aux usages informels de l'ethnicité dans le fonctionnement quotidien des États, et les faux-semblants du débat entre jacobinisme et multiculturalisme.

49. Les cérémonies (mariages ou enterrements de personnalités, intronisation d'un chef d'État, meetings politiques, passations de pouvoir entre ministres, campagnes

électorales, etc.) sont des lieux privilégiés de manifestation de ces alliances à plaisanterie. Ainsi lors du doua du $7^{e}$ jour après l'enterrement de l'ancien président Lamizana au Burkina ; « les Mossi ne cessaient guère de taquiner les Samos (ethnie du défunt) et ces derniers non plus ne cessaient de répliquer. On entendait par exemple certains dire qu'ils sont venus à la fête des Samos. D'autres faisaient des prémonitions pour dire que d'autres Samos (au moins une dizaine) rejoindront bientôt le Général. Et, les Samos de contester tout en soutenant le contraire. Pendant que tout cela se disait, sans rire, trois femmes débarquèrent sur les lieux avec un chien de pelage roux. Habillée dans une tenue de la culture moaga et l'autre d'un pagne à l'effigie du général confectionné sans doute à l'époque où il était aux affaires, ces femmes affirmaient avec un air sérieux, qu'elles sont venues offrir un taureau (le chien) pour la fête du vieux samo. Et une hilarité s'empara de la foule » (Bendré, 5 juin 2005). De même, au Mali, « au Palais des congrès, le chef de l'État Amadou Toumani Touré, le président de l'Assemblée nationale, Ibrahim Boubacar Keïta, et le ministre de la Culture, Cheikh Oumar Sissoko, tous des "sinankoun" avaient échangé des calebasses pleines de haricots en direct à la télévision nationale. La scène, appréciée du public, donnait une leçon importante en politique : on peut militer dans des chapelles différentes et rester des frères » (L'Essor, 27 février 2004). Au Sénégal enfin, lors d'un meeting politique du Premier ministre dans sa ville de Fatick, on a vu le Premier ministre (Macky Sall, Toucouleur), le président du Conseil régional (Ablaye Sène, Serer) et le représentant du MFDC (Bertrand Diamacoune, Joola) «se refiler » tour à tour le mouton et le sac de haricots, « cadeaux » offerts par la délégation du Fuuta, provoquant l'hilarité du public. É. SмITH, Carnet de terrain, 2 avril 2005.

50. Rapport du Mali, CERD/C/407/Add.2, § 156, Organisation des Nations unies, Comité pour l'élimination de la discrimination raciale, Genève, $61^{\mathrm{e}}$ session, séance du 21 août 2002. À la lecture du rapport présenté par le Mali, les membres du CERD réclament davantage d'informations sur le " cousinage ». Le rapporteur s'inquiète par ailleurs des possibilités de dérapages et de discriminations dans la pratique des senankuya. Le rapport du Mali et les séances de discussion sur ce rapport sont disponibles à l'adresse suivante : <http:// www.unhchr.ch/html/menu2/6/cerd/cerds61.htm - 61st>.

51. « Ô Mali, Ô peuple malien/Loin de toi/Tes valeurs m'accompagnent/Tes enseignements me réconfortent/[...]. Qui ose prétendre qu'il n'y a rien au Mali ? Venez avec moi venez découvrir la terre de mes ancêtres/Là où la "parenté à plaisanteries" opère des miracles/Entre gens de patronymes opposés/Entre beaux-frères et bellessœurs/Entre grands-parents et petits-enfants/Là où règne le respect mutuel/ô Mali qui a su réunir ses différents groupes/Autour de ces valeurs fondamentales [...]. » Chanson Niènafîng, Album Bowmboï, 2003, Label Bleu. Traduction bambara/français du livret. 52. «La Sanakuya n'est pas mauvaise en vérité, la Sanakuya n'est pas mauvaise/Il l'a dit, écoutons et acceptons que la Sanakuya n'est pas mauvaise [...]/Nos Ba, ne vous vexez pas, le monde est comme la sanakuya/[...] Eyoo mon cher, ma chère, la Sanakuya est faite pour plaisanter/La Sanakuya touche les femmes, la Sanakuya touche les hommes/La Sanakuya n'est pas faite pour nuire, la Sanakuya n'est pas faite pour ça/Dans les "quartiers" et les villes, qu'est-ce qu'elle est agréable !/Nos griots de Daaka, qu'est-ce qu'ils l'apprécient !/ 
Nos Diakhanké en Guinée, qu'est-ce qu'ils sont sympas (agréables) ! [...]/Eyoo chers amis, amusez-vous donc ! Eyoo, chers amis, la Sanakuya est la tradition/[...] Nos Ba, ne vous gonflez pas, "Super Diallo" arrive en personne ! [...] Diallo, nous sommes tous les mêmes ! Ba, nous sommes tous pareils! Nobles Barry, nous sommes tous les mêmes! Sow, nous sommes tous pareils! » Chanson Sanakuyagal, album éponyme, CDS Production, 2002. Traduit du pulaar par Mamadou Diallo.

53. Le clip (2006) accompagnant la chanson est une mise en scène de la communauté serer, notamment ses grands hommes (Senghor), agrémentée de clins d'œil aux cousins toucouleur et joola.

54. À prendre au sens propre, car Alain Sissao a, par exemple, réalisé une carte des « ethnies » alliées à plaisanterie au Burkina Faso, Raphaël Ndiaye ou Yoro Doro Diallo au Sénégal ont procédé à des recensions patronymiques pour établir la liste des alliances entre patronymes. L'ONG Enda a même matérialisé ces alliances et équivalences patronymiques en faisant porter des pancartes par des enfants avec un patronyme, montrant ses équivalents et alliés.

55. Le CELHTO a notamment numérisé un grand nombre d'émissions de radios rurales de divers pays d'Afrique de l'Ouest sur le thème des « alliances à plaisanterie » dans le cadre du projet ARTO.

56. Sacrée plaisanterie, film de David Helft et Ewa SANTAMARIA, La Nomade Production \& l'ORTM, s.d. [1993], 55', avec le célèbre acteur Bala Moussa Keïta.

57. Du coq à l'âme, Graphoui, ASBL, 1999, 53'. Les objectifs du film résument tout à fait le triple programme des entreprises de promotion de la parenté à plaisanterie : «Informer sur un particularisme africain peu connu. Porter un regard différent sur les conflits ethniques qui ravagent l'Afrique. Proposer une équivalence entre une donnée socioculturelle africaine et l'humanisme occidental du XVI ${ }^{\mathrm{e}}$ siècle. » Le livre éponyme (F ATON 1999) est né, nous dit l'auteur, d'une réflexion sur les conflits au Rwanda et Burundi. L'ouvrage met côte à côte des extraits d'entretiens sur la parenté à plaisanterie au Sénégal et au Burkina, des extraits de l'Éloge de la folie d'Érasme, et une lettre-témoignage d'un ami réfugié burundais sur la violence politique dans la région des Grands Lacs. Par cet agencement, l'auteur procède à une comparaison de la parenté à plaisanterie, "pratique millénaire d'Afrique ", avec l'humanisme d'Érasme qui utilisait les ressorts de la littérature populaire, du carnaval et de la bouffonnerie pour critiquer les puissants de son temps et les « guerres fratricides » de ses contemporains : «Comme Érasme, et bien avant son temps, les ethnies rivales d'Afrique de l'Ouest utilisent l'humour pour désamorcer la violence. »

58. À ne pas confondre, bien qu'elles se ressemblent, avec la Charte du Mande («1222») de la confrérie des chasseurs (CISSÉ \& KAMISSOKO 1991 : 39 ; CISSÉ, FOFANA \& SAGOT-D UVAUROuX 2003). La Charte de Kurukan Fuga («1236»), présentée comme « la première constitution démocratique de l'Empire médiéval du Mali » a été « restituée » par des traditionnistes manding lors d'un atelier sur les radios rurales tenu à Kankan en Guinée en mars 1998 sous l'égide de l'Agence internationale de la francophonie et Intermédia Consultants (coopération suisse). Les traditionnistes venus du Sénégal, du Mali et de la Guinée se sont réunis à huis-clos pour confronter leurs versions, puis le magistrat guinéen, Siriman Kouyaté, a organisé la traduction et produit une version écrite en 44 articles précédés d'un préambule afin d'en faire l'équivalent d'une constitution moderne. Tout comme la Charte du Mande, dont le calligraphe Aboubacar Fofana écrit que c'est un « trésor caché » (CISSÉ, FofANA \& SAGOT-DuvaurouX 2003), l'invention du « trésor » de la Charte de Kurukan Fuga, qui doit être publiée prochainement par l'Union africaine avec une 
préface de Djibril Tamsir Niane, est le fruit d'une véritable entreprise culturelle sousrégionale, en partenariat avec des ONG du nord, de codification et production de « textes » politiques africains. Avant même sa publication officielle, on note que certains manuels scolaires l'ont déjà intégré comme « grand texte fondateur » organisant la " citoyenneté de l'Empire », et qu'elle sert de support à des argumentations les plus diverses (décentralisation, démocratie locale, protection de l'environnement, féminisme, droit d'asile et immunités, droits de l'Homme, diversité culturelle, protection sociale, etc.) qui ont en commun de chercher et « inventer » dans le passé prestigieux des empires ouest-africains des équivalents de la Magna Carta (1215), du Bill of Rights $(1628,1689)$, de l' Habeas Corpus (1679) de la Déclaration des droits de l'Homme et du citoyen (1789) d'importation occidentale. L'inventeur de l'alphabet n'ko, Souleymane Kanté, avait déjà en son temps insisté sur le « partage du monde » et l'institutionnalisation des senankuya opérée par Sunjata Keïta lors de l'assemblée de Kurukan Fuga (AMSELLE 2001 : 198s) ainsi que Djibril Tamsir NiAnE (1960 : 138s). Dans la version « constitutionnelle » de la Charte (1998), « l'article 7 » stipule : « Il est institué entre les mandenka le sanankunya (cousinage à plaisanterie) et le tanamanyöya (forme de totémisme). En conséquence, aucun différend né entre ces groupes ne doit dégénérer, le respect de l'autre étant la règle. Entre beauxfrères et belles-sœurs, entre grands-parents et petits-enfants, la tolérance et le chahut doivent être le principe. »Charte disponible sur le site : <http://www.africa-orale.org/ charte.rtf>. Un calendrier de l'an 764 de Kurukan Fuga (an 2000 de l'ère chrétienne) a été édité et vendu lors du Salon du livre de jeunesse de Montreuil en 1999. Un film a également été produit par le réalisateur malien Alioune Ifra NDIAYE, Le Bal de Kurukanfuga, sur un spectacle conçu par BlonBa et l'ORTM autour de la proclamation de Kurukan Fuga. Le film a été l'élément central des émissions de l'ORTM pour le passage à l'an 2000.

59. Ainsi le programme de la $13^{e}$ édition de la Semaine nationale de la culture de 2006 prévoyait des séances quotidiennes de " joutes oratoires des parents à plaisanteries » place de la mairie à Bobo-Dioulasso.

60. Interview du Larlé Naaba Tigré sur la " parenté à plaisanterie », dans la revue française de coopération militaire et de défense Frères d'armes, $n^{\circ} 244,2004$.

61. Interview de Bakary Soumano sur le sujet.

62. Ainsi Djibril Tamsir Niane, Babacar Sedikh Diouf, Saliou SAmbou et Pathé Diagne, entre autres, ont commis des contributions centrées sur la parenté à plaisanterie lors de l'Atelier de réflexion Initiative de valorisation des capacités endogènes de prévention des conflits et de gouvernance, Conakry, 9-11 mars 2005, Club du Sahel et de l'Afrique de l'Ouest/OCDE, 2005. Mangoné NIANG a présenté une communication sur la Charte de Kurukan Fuga comme « mécanisme de gouvernance endogène pour la prévention des conflits », lors du Forum intergénérationnel sur la gouvernance endogène, Ouagadougou, 26-28 juin 2006, organisé par le Club du Sahel.

63. On note également que depuis 2004 l'association burkinabè Sagl-Taaba, qui sensibilise sur des questions d'éducation civique et de morale par le biais du théâtre, s'est donnée pour objectif de « redynamiser la parenté à plaisanteries au niveau national et dans les pays francophones de la sous-région; favoriser par ce fait l'intégration des peuples de l'Afrique de l'Ouest ; anéantir ou tout au moins amoindrir, grâce à l'intégration des peuples, les tensions interethniques et inter-étatiques ». Elle a notamment organisé une « nuit de la parenté à plaisanteries », voir Le Pays, n 3242, 29 octobre 2004.

64. Au Sénégal, un militant de l'Association culturelle Ageen et Diambogne déclare que « les jeunes ne connaissent pas tellement la portée de ce cousinage à plaisanteries. Mais avec la création de l'association Ageen Jamboñ nous sommes en train de les former »; 
entretien avec Yayha Badji, Ziguinchor, 29 novembre 2004. Au Burkina Faso, «l'Association pour la promotion de la parenté à plaisanterie veut réapprendre aux populations, surtout urbaines, l'essence de leur patrimoine culturel enfoui ou en voie de disparition » (SISSAO $2002:$ 155). Cette approche souligne à la fois la prégnance du schème tradition/modernité sur les représentations et la volonté d'en sortir. La référence au célèbre ouvrage de Cheihk Hamidou Kane, l'Aventure ambiguë, tant chez Alain SISSAO (2002 : 132) que chez Raphaël NDIAYE (1999), par exemple, n'est sans doute pas anodine. En 1961, Cheikh Hamidou KANE (1961 : 44) posait cette célèbre question à propos des enfants scolarisés dans l'École française : « Ce qu'ils apprendront vaut-il ce qu'ils oublieront? » et répondit en 1995 : «La réponse est qu'il est possible d'apprendre sans oublier, et même d'apprendre à nouveau ce qui a été oublié! » (KANE 1995 : 145); voir, sur ce point, NDIAYE (1999).

65. On note qu'ont existé, par le passé, des fêtes centrées sur les alliances à plaisanterie ou le cousinage, donnant souvent lieu à des jeux guerriers (des bastonnades ou coursepoursuite) entre alliés à plaisanterie, comme lors de la fête du Nouvel An musulman ( achoura en arabe, jombende en malinké, wowo en hausa) observée à Kankan en Guinée (H UMBLOT 1918 : 532) au Bondoukou (TAUXIER 1921 : 289) et au nord Nigéria (WILSON-H AFFENDEN 1930 : 125). Ces pratiques festives de bastonnades entre senankuw (et entre classes d'âges) lors du Nouvel An, également mentionnées par LABOURET (1934 : 102), P ERSON (1968 vol. 1 : 79) ou SCHILDKROUT (1978: 154) existent toujours à Kouroussa ou Siguiri en Guinée par exemple (KouYATÉ $2003: 74)$.

66. Par exemple au Sénégal, les travaux de Raphaël Ndiaye ont été repris et adaptés par l'ONG Gra-Redep et la direction de l'École élémentaire dans les manuels d'éducation civique du projet pilote de " gouvernement d'école » : descriptions, exemples, saynètes à jouer par les élèves qui doivent utiliser le cousinage pour résoudre un conflit au sein de l'école, Charte de Kurukan Fuga en annexe (DEE-GRA-REDEP 2003).

67. Les entreprises de promotion des alliances à plaisanterie développent un discours moral et moralisateur se basant sur les « valeurs du cousinage ", que celles-ci soit rebaptisées « éthique » (Diouf 1997), « code de conduite » (DoumBIA 2002) ou " doctrine » (voir cet article de presse tanzanien soulignant combien les populations urbanisées ne connaissent plus la « doctrine utani » : « Utani hailed for enhancing national unity », Sunday Observer, 2002, s.d.). Voir également la contribution d'Étienne SMITH dans ce volume.

68. Fraternité Matin, 17 novembre 2005.

69. Babacar Sédikh Diouf, cité dans FATON (1998: 10).

70. <http://www.jeux2005.ne/societe.htm>. Voir les contributions de Cécile CANUT, Marie-Aude FouÉRÉ, Sten HAGBERG, Étienne SMITH dans ce volume, pour l'analyse de ces divers discours de promotion.

71. <http://www.ambamali-jp.org/en/e01-01.html>.

72. «La parenté à plaisanterie est un sujet d'expertise nationale, élément d'un phénomène transnational pouvant servir utilement la paix dans la sous-région ». Boniface Batiana, président de l'AB3P, interview de Gonzague Rambaud « Injures diplomatiques! », Le Nouvel Afrique Asie, $\mathrm{n}^{\circ} 180$, septembre 2004. Au nombre des objectifs de l'AB3P au Burkina Faso, on note : « La mise en œuvre de la parenté à plaisanteries comme une expertise du Burkina Faso en matière de gestion des conflits » (SISSAO 2002 : 155). 
73. Les sites Internet et guides touristiques sont trop nombreux pour être cités ici, on peut se contenter d'un exemple zambien pour une présentation des joking relationships comme trait marquant du pays au même titre que ses paysages et sa faune.

74. Ainsi, le philosophe Jean-Louis SAGOT-Duvauroux dans sa « Lettre au consul de France » (L'Humanité, 31 octobre 2002) propose d'en faire la base de nouvelles relations entre ex-colonisateurs et ex-colonisés tout comme les senankuya aménageaient autrefois les relations entre vaincus et vainqueurs, ou bien l'anthropologue Thomas Hylland E RIKSEN : « This wonderful African institution deserves being exported to other continents. Perhaps Jews and Palestinians, or Christians and Muslims, might want to give it a try? ", Risking security, Paradoxes of social cohesion, Thomas Hylland Eriksen Inaugural lecture, Vrije Universiteit Amsterdam, 15 mars 2005.

75. Discours du 11 septembre 2003. Kélétigui MARIKO (1990:36) ne dit pas autre chose à propos du Niger : «L'unité nationale au Niger a devancé la création de l'État et repose sur les relations de parenté à plaisanteries entre les dix groupes ethnolinguistiques [...]. » De même au Sénégal, le Colloque sur les convergences culturelles de 1994 constatait que « l'unité culturelle profonde de la nation n'est pas une construction politique mais le fruit de brassages séculaires opérés dans des foyers de convergences tels que le Fouta et le Gabu. Ceci démontre la possibilité au plan africain, d'une citoyenneté transcendant l'appartenance ethnique et instituant la diversité comme étape d'une richesse, et non comme une fin » (TAMBADOU $1996: 36)$.

76. Discours du Premier ministre Mohamed Ag Hamani à la Conférence épiscopale de l'Afrique de l'Ouest francophone, 4 février 2003. La phrase complète est la suivante : «Carrefour de civilisations, le Mali est une nation héritière d'illustres empires et royaumes, qui ont façonné son destin par un brassage multiséculaire transformant partout sa diversité en ficelles relationnelles que chaque Malien est fier d'assumer par le biais du cousinage à plaisanterie dont les répliques aromatisées sont des délices de l'acception mutuelle. »

77. La presse américaine, à la recherche d'une démocratie dans le monde islamique et d'un multi-ethnisme pacifique en Afrique, reprend à son compte l'idée des senankuya comme facteur explicatif de l'exception malienne qui semble tant l'émerveiller : "Seeing Mali », Washington Post, 20 mai 2001 ; «Democratic Mali Sets an Example », State Magazine, avril 2003, n 467, 10-15; « Polling Timbuktu : Islamic Democracy? Mali Finds a Way to Make It Work », The Wall Street Journal, 22 juin 2004 ; « Miracle in Mali », The Wilson Quaterly, 31 juillet 2006. Pour une analyse de la décentralisation au Mali et la mobilisation de récits historiques de légitimation, loin des éloges appuyés des journalistes, voir A MSELLE (2004) et FAY (dans ce volume).

78. Ainsi, par exemple, dans l'argumentaire du document officiel établissant les priorités thématiques de la recherche au Burkina Faso, le génocide rwandais est expressément mentionné comme contre-modèle soulignant l'urgence d'une réflexion sur la " gestion » des « relations interethniques » et la nécessité d'enquêtes sur les « parentés à plaisanteries » (CNRST 1995 : 361). Il en va de même dans l'ouvrage sur les Convergences culturelles au Sénégal reprenant le colloque de juin 1994 (TAMBADOU 1996).

79. On remarque que la prégnance des stéréotypes ethnistes sur l'Afrique est telle que le conflit et le «tribalisme » sont posés comme résultat normal et attendu de toute diversité « ethnique ». C'est donc l'absence de conflit ethnique qui relèverait alors du « miracle », de la « chance » et de « l'exception »... Ainsi, sous forme lapidaire, le sous-titre de cet article sur la parenté à plaisanterie au Burkina Faso : « Soixante-deux ethnies, zéro 
conflit ! », (« Parenté à plaisanterie : moquez-vous mais ne vous battez pas ! », op. cit.). De même dans Mond'Info, op. cit., le journal de l'ONG Enfants du Monde, on lit dans un article, toujours à propos de la parenté à plaisanterie au Burkina Faso : « La paix sociale qui y règne semble en contradiction avec le caractère composite et hétérogène de son peuple » (nos italiques).

80. En insistant sur ce qui lie, on valide par là même les catégories qui doivent être reliées. L'aporie du métissage, qui postule au préalable la distinction d'essences pures à mélanger (AMSELLE 1990), n'est pas facilement résolue dans ce discours d'une communauté nationale faite de la coalition d'éléments eux-mêmes métissés. Bien que généralement dirigé contre le récit de l'autochtonie (en dehors de la valorisation d'une «tradition africaine » vis-à-vis du reste du monde), le récit de promotion des alliances à plaisanterie peut parfois aller de pair avec celui-ci. Ainsi le professeur ivoirien Henri Legré célèbre-t-il les alliances à plaisanterie comme facteur d'unité en Côte-d'Ivoire... mais pour mieux souligner « l'intrusion d'éléments exogènes » : « La Côte-d'Ivoire, bien avant la colonisation, a mis en place des matériaux de la conscience nationale préétablie. Je souligne par là la "parenté ou les parentés à plaisanterie". Nous avons quatre ceintures de parenté, quatre axes de "parentés à plaisanterie", depuis les 18 montagnes jusque chez les Lobi. Tous les peuples qui se trouvent dans cette ceinture, sont des parents. [...] elle a fait en sorte que tous ceux qui se considèrent comme des parents, et par ricochet, les alliés des alliés, ne puissent jamais en venir aux mains, encore moins s'entretuer. C'est la raison pour laquelle pendant très longtemps, on a connu une stabilité relative, et c'est ce qui témoigne d'ailleurs de l'intrusion d'éléments exogènes dans le champ politique ivoirien pour le désorganiser », L'Inter, $n^{\circ}$ 1533, 19 juin 2003.

81. On notera que l'imagination impériale rétrospective de l'empire du Gabu donne aussi lieu à des réappropriations plus autochtonistes, contre les États-nations existants, par exemple dans le discours du MFDC en Casamance ou que l'appropriation de l'héritage des empires du Mali et du Wagadou peut donner lieu à controverses entre États (Mali vs Guinée, etc.).

82. La tension entre l'universalité ou la spécificité des remèdes aux conflits habite à la fois les acteurs internationaux et les acteurs locaux.

83. On peut cependant se demander si ce discours n'édifie pas des frontières internes entre Afrique de l'Ouest et Afrique centrale, voire entre peuples sahéliens et « peuples de la forêt ", reprenant à son compte une dichotomie raciale trop bien connue... En 1950, Fily Dabo SIssоко (1950 : 230) écrivait en ce sens : «Il [le senankuya] s'observe dans presque toutes les races soudanaises lesquelles, parait-il, se différencient par là des races bantoues qui, par ailleurs, ignorent les castes. » C'est aussi le point de vue actuel d'un journaliste américain, Yaroslav Trofimov : « That tradition is known to locals as "cousinage", and arose as a way to preserve peace as empire succeeded empire in medieval times. The descendants of winners and losers were usually made "cousins" in order to bury grievances. The tradition of loyalty to multiple cousin groups still defines social relationships in Mali, in contrast to the tribal allegiances that are the rule in much of the Arab world and tropical Africa further south », Wall Street Journal, 22 juin 2004 (nos italiques). On remarque à l'inverse que dans les travaux sur la Tanzanie, par exemple, il est souligné que les relations d'utani existeraient uniquement chez, et entre, les « tribus bantoues » (sic) (CHRISTENSEN $1963: 1324)$.

84. Cette double mise en scène des alliances à plaisanterie comme « chance » et spécificité africaine d'une part et exemple à suivre pour d'autres contrées d'autre part, se retrouve dans la plupart des articles de presse : « Lettre à Monsieur le consul de France au Mali de 
J.-L. Sagot-Duvauroux », L'Humanité, 31 octobre 2002 ; « Utani hailed for enhancing national unity », Sunday Observer, 2002, n.p. ; " Joking with tribalism », News from Tanzania, 2003 (1) ; « Parenté à plaisanterie : moquez-vous mais ne vous battez pas ! », Syfia International, $1^{\mathrm{er}}$ septembre 2001 ; «La parenté à plaisanterie : véritable régulateur social au Burkina Faso ", Mond'Info, $\mathrm{n}^{\circ} 1$, mars 2004, p. 2 ; « Le revenir de la parenté à plaisanterie : alliance, catharsis, régulateur social », Afrikara, 13 juillet 2004 ; « Injures diplomatiques ! ", Le Nouvel Afrique Asie, $\mathrm{n}^{\circ}$ 180, sept. 2004 ; « Une drôle de tradition », La Grande Époque, 12 juillet 2005.

85. L'« invention » de La Charte de Kurukan Fuga et la promotion de la parenté à plaisanterie en Afrique de l'Ouest semblent susciter en Afrique centrale des entreprises mimétiques de recherche de «textes fondateurs » analogues à celui de Kurukan Fuga et d'institutions comparables à la parenté à plaisanterie. Ainsi, la « déclaration de Libreville » de 2003 concluant la « conférence sur le dialogue interculturel », tenue sous l'égide de l'UNESCO et de la Francophonie recommande chaudement : « Le renforcement d'espaces publics et la création de nouveaux espaces permettant l'évacuation de l'agressivité sur le modèle de la "parenté à plaisanterie". [...] l'encouragement d'études systématiques des épopées et d'autres grands textes fondateurs de la région à l'instar des études sur l'épopée mandingue. La redynamisation du Centre international des civilisations Bantu (CICIBA) et l'achèvement de la construction de son siège national » (UNESCO 2005 : 21) (nos italiques).

86. Dans la littérature savante, le débat sur les origines des alliances à plaisanterie a notamment porté sur la question de l'antériorité, ou non, des parentés à plaisanterie intrafamiliales, sur les alliances interclaniques et interethniques. C'est-à-dire sur l'extension possible, par mimétisme, des relations avunculaires et de cousinage aux relations entre différentes communautés politiques, l'appréhension de l'extériorité, l'incorporation des étrangers et l'entretien de relations politiques se faisant progressivement, dans un idiome hiérarchique familier, celui de la famille. Pour $C$ HRISTENSEN (1963 : 1324), comme pour MOREAU (1941: 5), le passage s'est fait par un processus d'extension de l'utani interne (intrafamilial, puis intraclanique) à l'utani externe (interethnique). À l'inverse, pour GRIAULE (1948 : 242-243, 255), le mangou entre Bozo et Dogon serait la véritable « institution originelle », les rapports mangou entre différents groupes dogon n'étant que des institutions dérivées et mimétiques du mangou Dogon-Bozo. On ne prétend pas résoudre cette question ici, mais simplement souligner que l'alliance interethnique est vraisemblablement la dernière venue, contemporaine des processus discursifs d'ethnogenèses (précoloniales, coloniales et postcoloniales), et surtout que la question est mal posée. Distinguer de façon radicale, comme le fait Moreau, entre une relation « interne » (intratribal utani) au groupe et une relation externe (intertribal utani) revient à poser comme déterminante la frontière du groupe alors que la similarité des relations plaide au contraire en faveur d'un continuum. D'ailleurs l'énonciation et la répétition de la relation contribuent largement à pr 


\section{AUTEURS}

\section{CÉCILE CANUT}

MoDyCo-CNRS-Paris X-Nanterre.

\section{ÉTIENNE SMITH}

Centre d'études et de recherches internationales, IEP, Paris. 